IFUM 426/FT

hepth@xxx/9207018

\title{
QUANTUM CONSERVED CURRENTS IN SUPERSYMMETRIC TODA THEORIES
}

\author{
A. GUALZETTI, S. PENATI and D. ZANON \\ Dipartimento di Fisica dell'Università di Milano and \\ INFN, Sezione di Milano, Via Celoria 16, I-20133 Milano, Italy
}

\begin{abstract}
We consider $N=1$ supersymmetric Toda theories which admit a fermionic untwisted affine extension, i.e. the systems based on the $A(n, n), D(n+1, n)$ and $B(n, n)$ superalgebras. We construct the superspace Miura trasformations which allow to determine the $\mathrm{W}$-supercurrents of the conformal theories and we compute their renormalized expressions. The analysis of the renormalization and conservation of higher-spin currents is then performed for the corresponding supersymmetric massive theories. We establish the quantum integrability of these models and show that although their Lagrangian is not hermitian, the masses of the fundamental particles are real, a property which is maintained by one-loop corrections. The spectrum is actually much richer, since the theories admit solitons. The existence of quantum conserved higherspin charges implies that elastic, factorized S-matrices can be constructed.
\end{abstract}

IFUM 426/FT

June 1992 


\section{Introduction}

Whenever fermionic fields can be coupled consistently to their bosonic partners, it is of interest to consider the supersymmetric version of the theory. In particular this can be done for a class of two-dimensional models known as Toda theories. The bosonic systems are constructed from a Lie algebra and depending on whether the algebra is affine or not, the resulting theories are massive or conformally invariant, respectively. They possess an infinite number of conservation laws which are not spoiled by quantum anomalies [1], 2, 3]. This implies the existence of factorized, elastic S-matrices [4], which have been determined exactly for all massive theories based on simply-laced [5] as well as nonsimply-laced algebras [6].

Fermions can be added [7] while maintaining the basic property of classical integrability: in this case one has to construct a field theory starting from a Lie superalgebra [8]. In general the introduction of fermions does not lead to systems invariant under supersymmetry transformations; indeed all unitary fermionic Toda theories exhibit explicitly broken supersymmetry [7]. In order to obtain a supersymmetric model one has to consider those superalgebras which admit a purely fermionic simple root system. In this paper we focus on these theories and among them we select the ones for which the untwisted affine perturbation can be implemented also in a supersymmetric way. Leaving aside the exceptional superalgebras $D(2,1 ; \alpha)$, we consider the $A(n, n), D(n+1, n)$ and $B(n, n)$ supersymmetric Toda theories and address the issue of their quantum integrability. We show that both in the conformal and in the massive cases there exist quantum higher-spin conserved currents. In the massive cases this ensures the commutativity of the corresponding charges with the S-matrix; thus the scattering is purely elastic and factorizes into two-body processes. At the Lagrangian level these theories are nonunitary, nonetheless the particle and soliton mass spectrum is real and the explicit construction of exact S-matrices could be attempted.

In Section 2 we briefly review basic notions about superalgebras and set the $N=1$ superspace notation. Then we construct the classical Miura operators for the above mentioned supersymmetric theories. The specific examples of the $D(2,1), B(1,1)$ and $A(1,1)$ conformal theories are presented in Section 3, where we obtain the corresponding classical W-supercurrents and compute their renormalized expressions. In Section 4 we consider the fermionic affine extension of these models and we explicitly construct the first higher-spin conserved currents to all orders of perturbation theory. Finally in Section 5 we compute the particle mass spectra up to one-loop for the $A^{(1)}(n, n), D^{(1)}(n+1, n)$ and $B^{(1)}(n, n)$ theories with $n=1,2$. In accordance with previous results for unitary models, we find that in the $A$ and $D$ series, being the roots all of the same length (simply-laced), the classical mass ratios are maintained at the quantum level, while for the nonsimply-laced $B$-theories this is not true. Section 6 contains some final remarks 
and our conclusions. In the Appendix we have collected further details on the choice of the fermionic roots and of the corresponding generators and explicitly constructed the weights of the fundamental representation for the various superalgebras.

\section{$2 \mathrm{~N}=1$ supersymmetric Toda theories}

Two-dimensional integrable theories with $N=1$ supersymmetry can be constructed as Toda models based on a purely fermionic root system of a Lie superalgebra [7].

A rank $r$ Lie superalgebra $\mathcal{G}=\mathcal{G}_{0}+\mathcal{G}_{1}$ is a graded algebra whose Lie bracket is defined as $[a, b]=a b-(-1)^{\operatorname{deg}(a) \operatorname{deg}(b)} b a$, where $\operatorname{deg}(a)=0$ if $a \in \mathcal{G}_{0}$ and $\operatorname{deg}(a)=1$ if $a \in \mathcal{G}_{1}$. The generators $\vec{h}, e_{i}^{+}, e_{j}^{-}$satisfy the graded commutation relations

$$
[\vec{h}, \vec{h}]=0 \quad\left[\vec{h}, e_{j}^{ \pm}\right]= \pm \vec{\alpha}_{j} e_{j}^{ \pm} \quad\left[e_{i}^{+}, e_{j}^{-}\right\}=\delta_{i j} \vec{h} \cdot \vec{\alpha}_{i}
$$

where $\vec{h}$ are the $r$ generators of the Cartan subalgebra and $e_{j}^{ \pm}$are the basis vectors associated to the simple roots $\vec{\alpha}_{j}$, with $j=1, \ldots, r$. Depending on whether $e_{j}^{ \pm}$is an element of $\mathcal{G}_{0}$ or of $\mathcal{G}_{1}, \vec{\alpha}_{j}$ is referred to as an even or odd root, respectively.

Lie superalgebras have been classified by Kač [8] on the basis of the associated Cartan matrix. A distinguishing feature is given by the fact that the system of simple roots (i.e. the Dynkin diagram) is not unique (up to a Weyl transformation). In general it is possible to define several unequivalent sets which have a different content of fermionic roots: they are related by generalized Weyl transformations associated to fermionic roots [9].

A classical Lie superalgebra $\mathcal{G}$ can be suitably extended and a new infinite dimensional one emerges, the affine Lie superalgebra. In particular the untwisted affine extension $\mathcal{G}^{(1)}$ is realized by adding to a system of simple roots of $\mathcal{G}$ the lowest root defined as

$$
\vec{\alpha}_{0}=-\sum_{i=1}^{r} q_{i} \vec{\alpha}_{i}
$$

where $q_{i}$ are the Kač labels. In the following we will focus on untwisted affine Lie superalgebras which admit a purely fermionic extended set of simple roots. They are the untwisted extensions of:

1) the unitary series $A(n, n) \equiv \operatorname{sl}(n+1, n+1 ; \mathcal{C}), n \geq 1$. The superalgebra $A(n, n)$ is not simple; it possesses a one-dimensional graded invariant subalgebra generated by the basis element $\mathbf{I}_{2 n+2} . A(n, n)$ is then defined as $\operatorname{sl}(n+1, n+1 ; \mathcal{C})$ factored by this subspace. 2) The orthosymplectic series $D(n+1, n) \equiv O \operatorname{sp}(2 n+2,2 n ; \mathcal{C}), n \geq 1$ and $B(n, n)=$ $O \operatorname{sp}(2 n+1,2 n ; \mathcal{C}), n \geq 1$. These superalgebras are simple.

3) Among the exceptional superalgebras only $D(2,1 ; \alpha), \alpha \neq 0,-1$, have a fermionic Dynkin diagram with the lowest root also fermionic. They are deformations of the $D(2,1)$ superalgebra. 
We restrict our analysis to the series listed in 1) and 2).

The classical field equations for a supersymmetric Toda theory based on a Lie superalgebra can be viewed as the zero curvature condition on a Lax connection in $N=1$ superspace $(z, \bar{z}, \theta, \bar{\theta})$, where $z, \bar{z}$ are Minkowski space light-cone coordinates

$$
\begin{aligned}
& z \equiv x^{+}=\frac{1}{\sqrt{2}}\left(x^{0}+x^{1}\right) \quad \bar{z} \equiv x^{-}=\frac{1}{\sqrt{2}}\left(x^{0}-x^{1}\right) \\
& \partial \equiv \partial_{z}=\frac{1}{\sqrt{2}}\left(\partial_{0}+\partial_{1}\right) \quad \bar{\partial} \equiv \partial_{\bar{z}}=\frac{1}{\sqrt{2}}\left(\partial_{0}-\partial_{1}\right) \quad \square=2 \partial \bar{\partial}
\end{aligned}
$$

and $\theta, \bar{\theta}$ are the $(1,1)$ spinor coordinates. Introducing the corresponding covariant derivatives

$$
D=\partial_{\theta}+i \theta \partial \quad \bar{D}=\partial_{\bar{\theta}}-i \bar{\theta} \bar{\partial}
$$

satisfying the commutation relations $\{D, \bar{D}\}=0, D^{2}=i \partial, \bar{D}^{2}=-i \bar{\partial}$, and denoting by $\vec{\Phi}$ the superfields

$$
\Phi^{a}=\phi^{a}+\frac{1}{\sqrt{2}} \theta \psi^{a}+\frac{1}{\sqrt{2}} \bar{\theta} \bar{\psi}^{a}+\theta \bar{\theta} F^{a} \quad a=1, \ldots, r
$$

the Lax connection is a Lie superalgebra-valued gauge superfield [7, 10]

$$
U=D \vec{\Phi} \cdot \vec{h}-\lambda \sum_{j=0}^{r} e_{j}^{+} \quad \bar{U}=-\frac{1}{2 \lambda} \sum_{j=0}^{r} q_{j} e^{\vec{\alpha}_{j} \cdot \vec{\Phi}} e_{j}^{-}
$$

which is flat, i.e. it satisfies

$$
\bar{D} U+D \bar{U}+\{U, \bar{U}\}=0
$$

In eq.(2.6) we have introduced the spectral parameter $\lambda$ and chosen the principal gradation for the generators.

It is straightforward to check using the commutation relations in eq.(2.1) that the flatness condition in eq.(2.7) coincides with the equations of motion derivable from the supersymmetric Toda action

$$
S=\frac{1}{\beta^{2}} \int d^{2} z d^{2} \theta\left[D \vec{\Phi} \cdot \bar{D} \vec{\Phi}+\sum_{i=0}^{r} q_{i} e^{\vec{\alpha}_{i} \cdot \vec{\Phi}}\right]
$$

where $d^{2} z=d z d \bar{z}, d^{2} \theta=\bar{D} D$ and $\beta$ is the coupling constant. In order to obtain a realization of the supersymmetric massive systems based on the affine superalgebras $D^{(1)}(n+1, n), B^{(1)}(n, n)$ and $A^{(1)}(n, n)$ one substitutes in eq.(2.8) the explicit expressions of the fermionic roots given in the Appendix. The conformally invariant Toda theories are obtained correspondingly by dropping the exponential term containing the lowest root $\vec{\alpha}_{0}$.

At the classical level supersymmetric Toda field theories are integrable. The existence of an infinite number of conservation laws is a consequence of the identification of the 
Toda field equations with eq.(2.7). The most practical way to construct explicitly the conserved supercurrents is via the Miura operator which is obtained, like in the bosonic case 111, 12, 2, 13], by considering the Lax pair associated to eq.(2.7)

$$
(D+U) \chi=0 \quad(\bar{D}+\bar{U}) \chi=0
$$

In order to describe the affine and the conformal cases on equal footing, it is convenient to multiply the generator $e_{0}^{+}$associated to the lowest root by a c-number $c$, so that setting $c=0(\lambda=1)$ we restrict our results to the conformal theory, whereas $c=1$ gives the corresponding formulae for the affine theory. The Miura operators for the $D(n+1, n)$ and $B(n, n)$ conformal cases have also been constructed in refs. [14].

We start by constructing the Miura operator for the $D(n+1, n)$ theory. Using the explicit expression for the generators given in eqs.(A.12,A.13,A.15) of the Appendix, the first Lax equation in eq. (2.9) can be written as

$$
(D+D \vec{\Phi} \cdot \vec{h}) \chi=\lambda \Lambda \chi
$$

where $(D+D \vec{\Phi} \cdot \vec{h})$ and $\Lambda \equiv\left(\sum_{j=1}^{2 n+1} e_{j}^{+}+c e_{0}^{+}\right)$are the $(4 n+2) \times(4 n+2)$ matrices

$$
\begin{aligned}
(D+D \vec{\Phi} \cdot \vec{h})= & \operatorname{diag}\left\{D+D \Phi_{1}, \cdots, D+D \Phi_{n+1}, D-D \Phi_{1}, \cdots, D-D \Phi_{n+1},\right. \\
& \left.D+i D \Phi_{n+2}, \cdots, D+i D \Phi_{2 n+1}, D-i D \Phi_{n+2}, \cdots, D-i D \Phi_{2 n+1}\right\}
\end{aligned}
$$

and

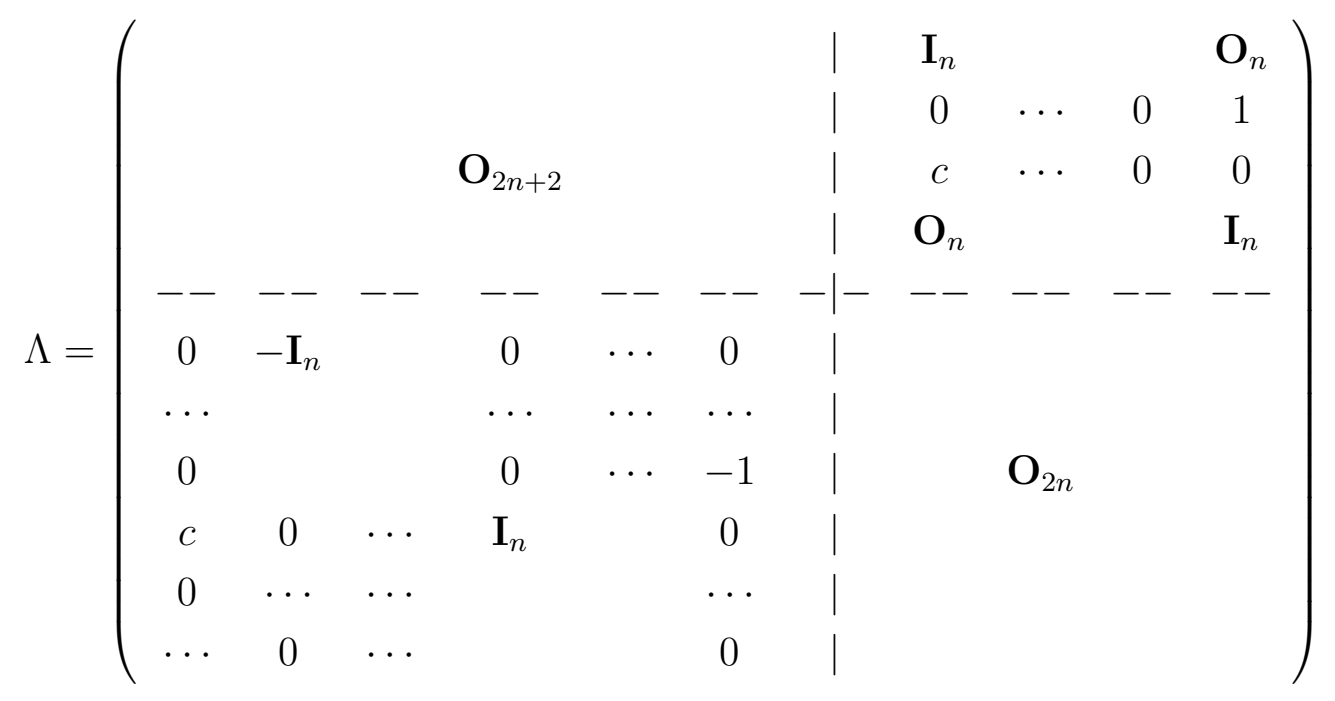

The vector $\chi$ in eq. (2.10) has $(4 n+2)$ superfield entries, the first $2 n+2$ obeying opposite statistics with respect to the others. Thus eq.(2.10) gives rise to the following set of coupled equations

$$
\left(D+D \Phi_{j}\right) \chi_{j}=\lambda \chi_{2 n+2+j} \quad j=1, \cdots, n
$$




$$
\begin{array}{rlrl}
\left(D+D \Phi_{n+1}\right) \chi_{n+1} & =\lambda \chi_{4 n+2} & \\
\left(D-D \Phi_{1}\right) \chi_{n+2} & =c \lambda \chi_{2 n+3} & \\
\left(D-D \Phi_{j}\right) \chi_{n+1+j} & =\lambda \chi_{3 n+1+j} & & j=2, \cdots, n+1 \\
\left(D+i D \Phi_{n+1+j}\right) \chi_{2 n+2+j} & =-\lambda \chi_{j+1} & j=1, \cdots, n-1 \\
\left(D+i D \Phi_{2 n+1}\right) \chi_{3 n+2} & =-\lambda\left(\chi_{n+1}+\chi_{2 n+2}\right) & \\
\left(D-i D \Phi_{n+2}\right) \chi_{3 n+3} & =\lambda \chi_{n+2}+c \lambda \chi_{1} & \\
\left(D-i D \Phi_{n+1+j}\right) \chi_{3 n+2+j} & =\lambda \chi_{n+1+j} & & j=2, \cdots, n
\end{array}
$$

It is easy to see that one can eliminate iteratively the $\chi_{2}, \cdots, \chi_{4 n+2}$ superfields and derive a single equation for $\chi_{1}$

$$
\Delta(\vec{\Phi}) \chi_{1}=4 c(-1)^{n} \lambda^{4 n} D \chi_{1}
$$

where

$$
\begin{aligned}
\Delta(\vec{\Phi})= & \left(D-D \Phi_{1}\right)\left(D-i D \Phi_{n+2}\right)\left(D-D \Phi_{2}\right)\left(D-i D \Phi_{n+3}\right) \cdots\left(D-i D \Phi_{2 n+1}\right) \\
& \left(D-D \Phi_{n+1}\right) \frac{1}{D}\left(D+D \Phi_{n+1}\right)\left(D+i D \Phi_{2 n+1}\right) \cdots\left(D+i D \Phi_{n+2}\right)\left(D+D \Phi_{1}\right)
\end{aligned}
$$

is the Miura operator for the $D(n+1, n)$ Toda theory. We note that $\Delta(\vec{\Phi})$ can be written equivalently in terms of the weights of the fundamental representation of the algebra (see eq.(A.17) in the Appendix)

$$
\begin{aligned}
\Delta(\vec{\Phi})= & \left(D+\vec{\lambda}_{4 n+2} \cdot D \vec{\Phi}\right)\left(D+\vec{\lambda}_{4 n+1} \cdot D \vec{\Phi}\right) \cdots \cdots\left(D+\vec{\lambda}_{2 n+2} \cdot D \vec{\Phi}\right) \\
& \frac{1}{D}\left(D+\vec{\lambda}_{2 n+1} \cdot D \vec{\Phi}\right)\left(D+\vec{\lambda}_{2 n} \cdot D \vec{\Phi}\right) \cdots\left(D+\vec{\lambda}_{1} \cdot D \vec{\Phi}\right)
\end{aligned}
$$

In this case, in complete analogy with the corresponding Miura operator for the bosonic Toda theory based on the $d_{n}$ Lie algebra [12, 13], $\Delta(\vec{\Phi})$ is a pseudo differential operator.

We specialize now the first Lax equation in (2.9) to the $B(n, n)$ system. Using the results listed in eqs.(A.19,A.20,A.22) of the Appendix, we have

$$
\begin{aligned}
(D+D \vec{\Phi} \cdot \vec{h})= & \operatorname{diag}\left\{D+D \Phi_{1}, \cdots, D+D \Phi_{n}, D-D \Phi_{1}, \cdots, D-D \Phi_{n}, D,\right. \\
& \left.D+i D \Phi_{n+1}, \cdots, D+i D \Phi_{2 n}, D-i D \Phi_{n+1}, \cdots, D-i D \Phi_{2 n}\right\}
\end{aligned}
$$


and $\Lambda=\sum_{j=1}^{2 n} e_{j}^{+}+c e_{0}^{+}$, that is

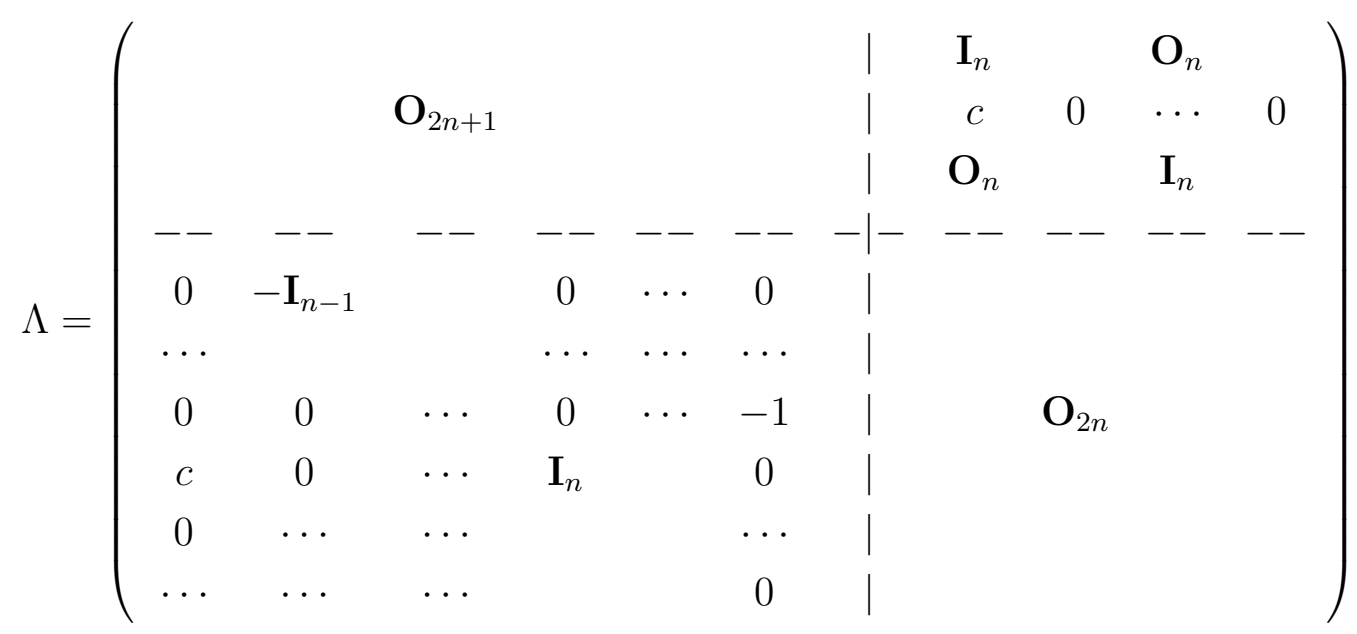

In this case the vector $\chi$ has the first $(2 n+1)$ components with opposite statistics with respect to the last $2 n$. The Lax equation is then equivalent to the system

$$
\begin{array}{rlrl}
\left(D+D \Phi_{j}\right) \chi_{j} & =\lambda \chi_{2 n+1+j} & & j=1, \cdots, n \\
\left(D-D \Phi_{1}\right) \chi_{n+1} & =c \lambda \chi_{2 n+2} & & j=2, \cdots, n \\
\left(D-D \Phi_{j}\right) \chi_{n+j} & =\lambda \chi_{3 n+j} & & \\
D \chi_{2 n+1} & =\lambda \chi_{4 n+1} & j=1, \cdots, n-1 \\
\left(D+i D \Phi_{n+j}\right) \chi_{2 n+1+j} & =-\lambda \chi_{j+1} & & \\
\left(D+i D \Phi_{2 n}\right) \chi_{3 n+1} & =-\lambda \chi_{2 n+1} & & \\
\left(D-i D \Phi_{n+1}\right) \chi_{3 n+2} & =\lambda \chi_{n+1}+c \lambda \chi_{1} & & \\
\left(D-i D \Phi_{n+j}\right) \chi_{3 n+1+j} & =\lambda \chi_{n+j} &
\end{array}
$$

from which $\chi_{2}, \cdots, \chi_{4 n+1}$ can be eliminated and we obtain

$$
\Delta(\vec{\Phi}) \chi_{1}=2 c(-1)^{n} \lambda^{4 n} D \chi_{1}
$$

where

$$
\begin{aligned}
\Delta(\vec{\Phi})= & \left(D-D \Phi_{1}\right)\left(D-i D \Phi_{n+1}\right)\left(D-D \Phi_{2}\right)\left(D-i D \Phi_{n+2}\right) \cdots\left(D-D \Phi_{n}\right) \\
& \left(D-i D \Phi_{2 n}\right) D\left(D+i D \Phi_{2 n}\right)\left(D+D \Phi_{n}\right) \cdots\left(D+i D \Phi_{n+1}\right)\left(D+D \Phi_{1}\right)
\end{aligned}
$$

The operator in eq.(2.21) generates the $W$-currents for the conformal $B(n, n)$ Toda field theory. It can be written as

$$
\Delta(\vec{\Phi})=\left(D+\vec{\lambda}_{4 n+1} \cdot D \vec{\Phi}\right)\left(D+\vec{\lambda}_{4 n} \cdot D \vec{\Phi}\right) \cdots \cdot\left(D+\vec{\lambda}_{2} \cdot D \vec{\Phi}\right)\left(D+\vec{\lambda}_{1} \cdot D \vec{\Phi}\right)
$$

where $\vec{\lambda}_{1}, \cdots, \vec{\lambda}_{4 n+1}$ are the weights of the fundamental representation of the algebra (see eq. (A.23)). 
Finally we consider the Miura transformation for the $A(n, n)$ case. The generators of the algebra obtained by factoring out the invariant subalgebra $\left\{\mathbf{I}_{2 n+2}\right\}$ are given in eqs.(A.24,A.25,A.26) of the Appendix. We have

$$
\begin{aligned}
(D+D \vec{\Phi} \cdot \vec{h})= & \operatorname{diag}\left\{D+D \Phi_{1}+\cdots+D \Phi_{n}+i D \Phi_{n+1}+\cdots+i D \Phi_{2 n},\right. \\
& D-D \Phi_{1}+D \Phi_{2}+\cdots+D \Phi_{n}+i D \Phi_{n+1}+\cdots+i D \Phi_{2 n}, \\
& D-D \Phi_{2}+D \Phi_{3}+\cdots+D \Phi_{n}+i D \Phi_{n+2}+\cdots+i D \Phi_{2 n}, \\
& \cdots \cdots, D-D \Phi_{n}+i D \Phi_{2 n}, \\
& D+D \Phi_{2}+\cdots+D \Phi_{n}+2 i D \Phi_{n+1}+i D \Phi_{n+2}+\cdots+i D \Phi_{2 n}, \\
& D+D \Phi_{3}+\cdots+D \Phi_{n}+2 i D \Phi_{n+2}+i D \Phi_{n+3}+\cdots+i D \Phi_{2 n}, \\
& \left.\cdots \cdots, D+2 i D \Phi_{2 n}, D\right\}
\end{aligned}
$$

and

$$
\Lambda=\sqrt{2}\left(\begin{array}{cc:ccc} 
& & & & \\
& \mathbf{O}_{n+1} & \mathbf{I}_{n+1} & \\
-- & -- & - & -- & -- \\
0 & \mathbf{I}_{n} & & \mathbf{O}_{n+1} & \\
c & 0 & \mid & &
\end{array}\right)
$$

The $(2 n+2)$-dimensional vector $\chi$ contains $(n+1)$ fermionic and $(n+1)$ bosonic superfields. The Lax equation $(D+D \vec{\Phi} \cdot \vec{h}) \chi=\lambda \Lambda \chi$ gives rise to the system

$$
\begin{array}{rlrl}
\left(D+\sum_{k=1}^{n} D \Phi_{k}+i \sum_{k=n+1}^{2 n} D \Phi_{k}\right) \chi_{1} & =\sqrt{2} \lambda \chi_{n+2} & \\
\left(D-D \Phi_{j}+\sum_{k=j+1}^{n} D \Phi_{k}+i \sum_{k=n+j}^{2 n} D \Phi_{k}\right) \chi_{j+1} & =\sqrt{2} \lambda \chi_{n+2+j} & & j=1, \cdots, n \\
\left(D+\sum_{k=j}^{n} D \Phi_{k}+2 i D \Phi_{n+j-1}+i \sum_{k=n+j}^{2 n} D \Phi_{k}\right) \chi_{n+j} & =\sqrt{2} \lambda \chi_{j} & j=2, \cdots, n+1 \\
D \chi_{2 n+2} & =c \sqrt{2} \lambda \chi_{1} &
\end{array}
$$

Again it can be reduced to

$$
\Delta(\vec{\Phi}) \chi_{1}=c(\sqrt{2} \lambda)^{2 n+2} \chi_{1}
$$

where

$$
\begin{aligned}
\Delta(\vec{\Phi})= & D\left(D-D \Phi_{n}+i D \Phi_{2 n}\right)\left(D+2 i D \Phi_{2 n}\right)\left(D-D \Phi_{n-1}+D \Phi_{n}+i D \Phi_{2 n-1}+i D \Phi_{2 n}\right) \\
& \cdots \cdots\left(D+D \Phi_{2}+\cdots+D \Phi_{n}+2 i D \Phi_{n+1}+i D \Phi_{n+2}+\cdots+i D \Phi_{2 n}\right) \\
& \left(D+D \Phi_{1}+\cdots+D \Phi_{n}+i D \Phi_{n+1}+\cdots+i D \Phi_{2 n}\right)
\end{aligned}
$$

gives the Miura operator for the $A(n, n)$ Toda field theory. This operator has the general form

$$
\Delta(\vec{\Phi})=\left(D+\vec{\lambda}_{2 n+2} \cdot D \vec{\Phi}\right)\left(D+\vec{\lambda}_{2 n+1} \cdot D \vec{\Phi}\right) \cdots \cdot\left(D+\vec{\lambda}_{1} \cdot D \vec{\Phi}\right)
$$


in terms of the weights of the fundamental representation of the superalgebra (see eq.(A.29)). We note that the results in eqs. $(2.22,2.28)$ are in agreement with the statement in Ref. [10].

From the knowledge of the differential operators in eqs. $(2.15,2.21,2.27)$ one easily obtains the currents of the conformal Toda theories. We construct them explicitly in the next section.

\section{$3 W$-supercurrents of the conformal theories}

As we have mentioned, the general action which describes the supersymmetric conformally invariant Toda field theories can be obtained from eq.(2.8) by neglecting the interaction term associated to the lowest root $\vec{\alpha}_{0}$. For such systems the existence of classically conserved higher-spin supercurrents follows from the fact that the Toda field equations are the integrability conditions of the linear problem in eq.(2.9). Indeed, in the previous section the first of these two equations has been used to construct the Miura operator $\Delta(\vec{\Phi})$ which, for the $B(n, n)$ and $A(n, n)$ theories, can be written as

$$
\Delta(\vec{\Phi})=D^{d}+\sum_{i=0}^{d-1} W^{\left(\frac{d-1}{2}-\frac{i}{2}\right)} D^{i}
$$

where $d$ is the dimension of the fundamental representation of the superalgebra. For the $D(n+1, n)$ theory the Miura operator in eq.(2.15) is non local and an identity like (3.1) holds for the local part. In addition, because of the $\frac{1}{D}$ factor, $d$ equals the dimension of the fundamental representation decreased by one.

For the $D(n+1, n)$ and $B(n, n)$ theories, using the Toda field equations, one can show that

$$
[\bar{D}, \Delta(\vec{\Phi})]=0
$$

Therefore the $W^{(s)}$ coefficients in eq.(3.1) provide a set of classical superholomorphic spin-s currents

$$
\bar{D} W^{(s)}=0
$$

which ensure the classical integrability of these Toda systems. For the $A(n, n)$ case the equation (3.2) is not valid, however the $W^{(1)}$ current satisfies (3.3). Since the $W^{(1)}$ current is proportional to the stress-energy tensor all the Toda theories under consideration describe $N=1$ superconformal models.

The renormalization of the conservation laws in (3.3) can be studied following the procedure described in Ref. 15. The calculation is performed using massless perturbation theory, which is best suited for an all-loop analysis [3]. We define the quantum Lagrangian by normal ordering the exponentials (no ultraviolet divergences), which are then treated as interaction terms. Potential anomalies in the quantum conservation laws would arise 
from local terms in

$$
\bar{D}_{Z}\left\langle W^{(s)}(Z, \bar{Z})\right\rangle \equiv \bar{D}_{Z}\left\langle W^{(s)}(Z, \bar{Z}) \exp \left(\frac{i}{\beta^{2}} \int d^{2} w d^{2} \theta^{\prime} \mathcal{L}_{i n t}\right)\right\rangle_{0}
$$

where $(Z, \bar{Z}) \equiv(z, \theta, \bar{z}, \bar{\theta})$. We introduce massless superspace propagators

$$
\left\langle\Phi_{i}(Z, \bar{Z}) \Phi_{j}(0,0)\right\rangle=-\delta_{i j} \frac{\beta^{2}}{4 \pi} \bar{D} D\left[\log (2 z \bar{z}) \delta^{(2)}(\theta)\right]
$$

and compute Wick contractions of the currents with the exponential in eq.(3.4). A spin$s$ current consists in a sum of terms with $2 s+1$ spinor derivatives acting on the fields. Standard superspace techniques allow to perform first the integration on the $\theta$-variable: in a given loop one reduces the number of covariant spinor derivatives from the various terms in the current and from the superspace propagators, using their commutation relations, to at most one $D$ and one $\bar{D}$ times a number of space-time derivatives. One obtains then a nonvanishing contribution if one ends up with exactly one $D$ and one $\bar{D}$, in which case

$$
\delta^{(2)}\left(\theta-\theta^{\prime}\right) \bar{D} D \delta^{(2)}\left(\theta-\theta^{\prime}\right)=\delta^{(2)}\left(\theta-\theta^{\prime}\right)
$$

Moreover since we are interested in determining only local contributions, it is sufficient to expand the exponential in eq.(3.4) to first order in $\mathcal{L}_{\text {int }}$. In fact, once the $D$-algebra has been performed we obtain terms of the form

$$
\bar{D}_{Z} \int d^{2} w \mathcal{A}(z, \bar{z}, \theta, \bar{\theta}) \bar{D}_{Z} \frac{1}{(z-w)^{n}} \mathcal{B}(w, \bar{w}, \theta, \bar{\theta})
$$

where $\mathcal{A}, \mathcal{B}$ are products of superfields and their $D$-derivatives. Now, using the prescription

$$
\bar{D}_{Z} \bar{D}_{Z} \frac{1}{(z-w)^{n}}=-i \bar{\partial}_{z} \frac{1}{(z-w)^{n}}=\frac{2 \pi}{(n-1) !} \partial_{w}^{n-1} \delta^{(2)}(z-w)
$$

we produce local contributions that we will try to cancel by renormalizing the classical $W^{(s)}$-currents, i.e. modifying them by coupling constant dependent terms. Thus for example, if we consider a typical contribution from a spin-1 current, like the energymomentum tensor, we obtain 


$$
\begin{aligned}
& \bar{D}_{Z}\left\langle D \Phi \partial \Phi\left(\frac{i}{\beta^{2}} \int d^{2} w d^{2} \theta^{\prime} e^{\Phi}\right)\right\rangle \\
& \sim i \bar{D}_{Z} \int d^{2} w\left[D \Phi \bar{D} \frac{-1}{4 \pi(z-w)} D e^{\Phi}+\partial \Phi \bar{D} \frac{i}{4 \pi(z-w)} e^{\Phi}-\frac{i \beta^{2}}{16 \pi^{2}} \bar{D} \frac{1}{(z-w)^{2}} e^{\Phi}\right] \\
& \sim \frac{i}{2} D \Phi D e^{\Phi}-\frac{1}{2} \partial \Phi e^{\Phi}-\frac{\beta^{2}}{8 \pi} \partial e^{\Phi}
\end{aligned}
$$

where in the last equality we have kept the first term, which actually vanishes, for pedagogical reasons.

In the next subsections we construct the $W$-currents for the simplest examples of superconformal Toda theories, namely the $D(2,1), B(1,1)$ and $A(1,1)$ theories. We compute first their classical expressions as derived from the Miura operator and obtain then their exact quantum corrected version.

\section{1) $\mathrm{D}(2,1)$ Toda theory}

The $D(2,1)$ superalgebra has rank 3 and we can express the fermionic simple roots as 3-dimensional vectors (cfr. eq.(A.12))

$$
\begin{aligned}
& \vec{\alpha}_{1}=\vec{\varepsilon}_{1}-\vec{\delta}_{1}=(1,0,-i) \\
& \vec{\alpha}_{2}=\vec{\delta}_{1}-\vec{\varepsilon}_{2}=(0,-1, i) \\
& \vec{\alpha}_{3}=\vec{\delta}_{1}+\vec{\varepsilon}_{2}=(0,1, i)
\end{aligned}
$$

The Toda action is written in terms of 3 scalar superfields

$$
S=\frac{1}{\beta^{2}} \int d^{2} z d^{2} \theta\left[D \vec{\Phi} \cdot \bar{D} \vec{\Phi}+e^{\Phi_{1}-i \Phi_{3}}+e^{-\Phi_{2}+i \Phi_{3}}+e^{\Phi_{2}+i \Phi_{3}}\right]
$$

It is symmetric under $\Phi_{2} \rightarrow-\Phi_{2}$; this will give rise to a corresponding definite parity of the currents.

Specializing the Miura operator in eq.(2.15) to the $n=1$ case we obtain

$$
\begin{aligned}
\Delta(\vec{\Phi}) & =\left(D-D \Phi_{1}\right)\left(D-i D \Phi_{3}\right)\left(D-D \Phi_{2}\right) \frac{1}{D}\left(D+D \Phi_{2}\right)\left(D+i D \Phi_{3}\right)\left(D+D \Phi_{1}\right) \\
& =D^{5}+\sum_{i=0}^{2} W^{\left(2-\frac{i}{2}\right)} D^{i}+F^{(1)} \frac{1}{D} F^{(1)}
\end{aligned}
$$

where we have defined

$$
F^{(1)} \equiv\left(D-D \Phi_{1}\right)\left(D-i D \Phi_{3}\right)\left(D-D \Phi_{2}\right) \mathbf{1}
$$

The local part of eq.(3.12) gives the following superholomorphic currents

$$
\begin{aligned}
W^{(1)}= & -i D \Phi_{1} \partial \Phi_{1}-i D \Phi_{2} \partial \Phi_{2}-i D \Phi_{3} \partial \Phi_{3}+2 i D \partial \Phi_{1}+D \partial \Phi_{3} \\
W^{\left(\frac{3}{2}\right)}= & -i \partial^{2} \Phi_{3}+i D \Phi_{1} D \partial \Phi_{1}-i D \Phi_{2} D \partial \Phi_{2}-\left(\partial \Phi_{3}\right)^{2}-i D \Phi_{3} D \partial \Phi_{3} \\
& +2 D \Phi_{1} D \partial \Phi_{3}-2 i D \Phi_{1} D \Phi_{2} \partial \Phi_{2}-2 i D \Phi_{1} D \Phi_{3} \partial \Phi_{3}+2 D \Phi_{2} \partial \Phi_{2} D \Phi_{3} \\
W^{(2)}= & \frac{1}{2} D\left(W^{\left(\frac{3}{2}\right)}+D W^{(1)}\right)
\end{aligned}
$$


whereas from the nonlocal term we obtain an additional spin-1 holomorphic current

$$
F^{(1)}=-i D \partial \Phi_{2}+i D \Phi_{1} \partial \Phi_{2}-i D\left(D \Phi_{2} D \Phi_{3}\right)+i D \Phi_{1} D \Phi_{2} D \Phi_{3}
$$

In particular $W^{(1)}$ can be written as

$$
W^{(1)}=-i D \vec{\Phi} \cdot \partial \vec{\Phi}+2 i \vec{\rho} \cdot D \partial \vec{\Phi}
$$

where $\vec{\rho}$ is the Weyl vector for the $D(2,1)$ superalgebra (see eq.(A.16) in the Appendix). It coincides with the improved stress-energy tensor of the theory.

We turn now to the study of the quantum extension of the classical conserved currents $W^{(1)}, F^{(1)}$ and $W^{\left(\frac{3}{2}\right)}$. We do not need to compute separately the renormalization of $W^{(2)}$ since it depends linearly on $W^{(1)}$ and $W^{\left(\frac{3}{2}\right)}$.

Inserting $W^{(1)}$ in eq.(3.4) and searching for potential anomalous terms, it is easy to check that in this case no anomalies appear, so that the classical expression in eq. (3.16) is not renormalized. This result agrees with the general statement [10] based on the study of the conformal dimensions of the interaction exponentials, according to which the quantum corrections to the stress-energy tensor can be obtained by a renormalization of the Weyl vector

$$
\vec{\rho}=\frac{1}{2} \sum_{i} \vec{\nu}_{i} \quad \longrightarrow \quad \vec{\rho}_{q}=\sum_{i} \frac{1}{2}\left(1+\frac{\beta^{2}}{4 \pi} \vec{\alpha}_{i}^{2}\right) \vec{\nu}_{i}
$$

where the vectors $\vec{\nu}_{i}$ are dual to the fermionic simple roots. Since in our case all the roots in eq.(3.10) have vanishing square, no renormalization is required. A simple calculation shows that also the $F^{(1)}$ current is not renormalized.

The corresponding analysis for the spin- $\frac{3}{2}$ current is somewhat more complicated. The most efficient way to compute the quantum corrections is to consider a linear combination of terms as in eq.(3.14) with arbitrary coefficients

$$
\begin{aligned}
W^{\left(\frac{3}{2}\right)}= & a \partial^{2} \Phi_{3}+b D \Phi_{1} D \partial \Phi_{1}+c D \Phi_{2} D \partial \Phi_{2}+d\left(\partial \Phi_{3}\right)^{2} \\
& +e D \Phi_{3} D \partial \Phi_{3}+f D \Phi_{1} D \partial \Phi_{3}+g D \Phi_{1} D \Phi_{2} \partial \Phi_{2} \\
& +h D \Phi_{1} D \Phi_{3} \partial \Phi_{3}+j D \Phi_{2} \partial \Phi_{2} D \Phi_{3}
\end{aligned}
$$

The unknowns $a, b, \cdots, j$ are then determined by requiring absence of anomalies in the quantum conservation law. To this end we proceed as described at the beginning of this section and compute all the local contributions which arise from Wick contracting the expression in eq.(3.18) with the interaction Lagrangian. We obtain $\left(\alpha \equiv \frac{\beta^{2}}{2 \pi}\right)$

$$
\begin{aligned}
& \bar{D}\left\langle W^{\left(\frac{3}{2}\right)}\left(\frac{i}{\beta^{2}} \int d^{2} w d^{2} \theta^{\prime} e^{\Phi_{1}-i \Phi_{3}}\right)\right\rangle \\
& \leadsto\left(\left[-\frac{1}{2} a+\frac{1}{2} b+\frac{i \alpha}{4} d-\frac{i}{2} f-\frac{\alpha}{4} h\right] D \Phi_{1} \partial \Phi_{1}\right.
\end{aligned}
$$




$$
\begin{aligned}
& +\left[\frac{i}{2} a-\frac{i}{2} b+\left(-1+\frac{\alpha}{4}\right) d-\frac{1}{2} f+\frac{i}{2}\left(-1+\frac{\alpha}{2}\right) h\right] D \Phi_{1} \partial \Phi_{3} \\
& +\left[-\frac{1}{2} a-\frac{1}{2} b+\frac{i \alpha}{4} d\right] D \partial \Phi_{1}+\left[\frac{i}{2} a+\frac{\alpha}{4} d+\frac{i}{2} e-\frac{1}{2} f\right] D \partial \Phi_{3} \\
& +\left[-\frac{1}{2} g-\frac{i}{2} j\right] D \Phi_{2} \partial \Phi_{2}+\left[\frac{1}{2} a+i\left(1-\frac{\alpha}{4}\right) d-\frac{1}{2} e+\frac{1}{2}\left(-1+\frac{\alpha}{2}\right) h\right] D \Phi_{3} \partial \Phi_{3} \\
& \left.+\left[\frac{i}{2} a+\frac{\alpha}{4} d-\frac{i}{2} e+\frac{i \alpha}{4} h\right] D \Phi_{3} \partial \Phi_{1}\right) e^{\Phi_{1}-i \Phi_{3}}
\end{aligned}
$$

and

$$
\begin{aligned}
& \bar{D}\left\langle W^{\left(\frac{3}{2}\right)}\left(\frac{i}{\beta^{2}} \int d^{2} w d^{2} \theta^{\prime} e^{\Phi_{2}+i \Phi_{3}}\right)\right\rangle \\
& \sim\left(\left[\frac{i}{2} a+\frac{i}{2} c+\left(1-\frac{\alpha}{4}\right) d-\frac{\alpha}{4} j\right] D \Phi_{2} \partial \Phi_{3}+\left[\frac{1}{2} a-\frac{1}{2} c+\frac{i \alpha}{4} d\right] D \partial \Phi_{2}\right. \\
& +\left[\frac{i}{2} f+\frac{1}{2}\left(1+\frac{\alpha}{2}\right) g-\frac{\alpha}{4} h\right] D \Phi_{1} \partial \Phi_{2}+\left[-\frac{1}{2} f+\frac{i \alpha}{4} g+\frac{i}{2}\left(1-\frac{\alpha}{2}\right) h\right] D \Phi_{1} \partial \Phi_{3} \\
& +\left[\frac{i}{2} a-\frac{\alpha}{4} d+\frac{i}{2} e-\frac{1}{2}\left(1+\frac{\alpha}{2}\right) j\right] D \Phi_{3} \partial \Phi_{2} \\
& +\left[\frac{1}{2} a+\frac{1}{2} c+\frac{i \alpha}{4} d+\frac{i}{2}\left(1+\frac{\alpha}{2}\right) j\right] D \Phi_{2} \partial \Phi_{2} \\
& +\left[-\frac{1}{2} a+i\left(1-\frac{\alpha}{4}\right) d-\frac{1}{2} e-\frac{i \alpha}{4} j\right] D \Phi_{3} \partial \Phi_{3}+\left[\frac{1}{2} g-\frac{1}{2} h\right] D \Phi_{1} D \Phi_{2} D \Phi_{3} \\
& \left.+\left[\frac{i}{2} a-\frac{\alpha}{4} d-\frac{i}{2} e\right] D \partial \Phi_{3}\right) e^{\Phi_{2}+i \Phi_{3}}
\end{aligned}
$$

The third exponential needs not be considered because of the $\Phi_{2}$-symmetry of the Lagrangian. The $W^{\left(\frac{3}{2}\right)}$ current will satisfy the conservation equation (3.3) if the coefficients of the various terms separately vanish. This leads to a set of equations for $a, b, \ldots, j$ which can be solved nontrivially. Thus we obtain, up to an overall normalization factor, the quantum spin- $\frac{3}{2}$ conserved current

$$
\begin{aligned}
W^{\left(\frac{3}{2}\right)}= & -i \partial^{2} \Phi_{3}+i\left(1-\frac{\beta^{2}}{4 \pi}\right) D \Phi_{1} D \partial \Phi_{1}-i\left(1+\frac{\beta^{2}}{4 \pi}\right) D \Phi_{2} D \partial \Phi_{2} \\
& -\left(\partial \Phi_{3}\right)^{2}-i\left(1+\frac{\beta^{2}}{4 \pi}\right) D \Phi_{3} D \partial \Phi_{3}+2 D \Phi_{1} D \partial \Phi_{3}-2 i D \Phi_{1} D \Phi_{2} \partial \Phi_{2} \\
& -2 i D \Phi_{1} D \Phi_{3} \partial \Phi_{3}+2 D \Phi_{2} \partial \Phi_{2} D \Phi_{3}
\end{aligned}
$$

Setting $\beta^{2}=0$ the classical current in eq.(3.14) is recovered.

\section{2) $\mathrm{B}(1,1)$ Toda theory}

For the case of the $B(1,1)$ superalgebra a convenient choice for the two simple roots is (cfr. eq.(A.19))

$$
\begin{aligned}
& \vec{\alpha}_{1}=\vec{\varepsilon}_{1}-\vec{\delta}_{1}=(1,-i) \\
& \vec{\alpha}_{2}=\vec{\delta}_{1}=(0, i)
\end{aligned}
$$


The corresponding supersymmetric Toda action is then given by

$$
S=\frac{1}{\beta^{2}} \int d^{2} z d^{2} \theta\left[D \Phi_{1} \bar{D} \Phi_{1}+D \Phi_{2} \bar{D} \Phi_{2}+e^{\Phi_{1}-i \Phi_{2}}+2 e^{i \Phi_{2}}\right]
$$

The classical $W$-algebra is obtained from the Miura operator in eq.(2.21) setting $n=1$. Explicitly we have

$$
\left(D-D \Phi_{1}\right)\left(D-i D \Phi_{2}\right) D\left(D+i D \Phi_{2}\right)\left(D+D \Phi_{1}\right)=D^{5}+\sum_{i=0}^{2} W^{\left(2-\frac{i}{2}\right)} D^{i}
$$

where

$$
\begin{aligned}
W^{(1)}= & -i D \Phi_{1} \partial \Phi_{1}-i D \Phi_{2} \partial \Phi_{2}+D \partial \Phi_{2}+2 i D \partial \Phi_{1} \\
W^{\left(\frac{3}{2}\right)}= & -i \partial^{2} \Phi_{2}+i D \Phi_{1} D \partial \Phi_{1}-\left(\partial \Phi_{2}\right)^{2}-i D \Phi_{2} D \partial \Phi_{2} \\
& +2 D \Phi_{1} D \partial \Phi_{2}-2 i D \Phi_{1} D \Phi_{2} \partial \Phi_{2} \\
W^{(2)}= & -D \partial^{2} \Phi_{1}+D \Phi_{1} \partial^{2} \Phi_{1}-i D \Phi_{1} \partial^{2} \Phi_{2}+i D \partial \Phi_{2} \partial \Phi_{1}-\left(\partial \Phi_{2}\right)^{2} D \Phi_{1} \\
& +\partial \Phi_{2} D \Phi_{2} \partial \Phi_{1}-i D \Phi_{2} D \partial \Phi_{2} D \Phi_{1} \\
= & \frac{1}{2} D\left(W^{\left(\frac{3}{2}\right)}+D W^{(1)}\right)
\end{aligned}
$$

We note that $W^{(1)}$ gives again the stress-energy tensor

$$
W^{(1)}=-i D \vec{\Phi} \cdot \partial \vec{\Phi}+2 i \vec{\rho} \cdot D \partial \vec{\Phi}
$$

where $\vec{\rho}$ is the Weyl vector (see eq.(A.16) in the Appendix).

In Ref. 15] we have shown that the holomorphic currents in eq.(3.25) maintain their form at the quantum level too, albeit the coefficients of the various terms acquire a coupling constant dependence. We report here the results and refer for details of the calculation to Ref.[15]. The quantum holomorphic stress-energy tensor is given by

$$
W^{(1)}=-i D \Phi_{1} \partial \Phi_{1}-i D \Phi_{2} \partial \Phi_{2}+\left(1-\frac{\beta^{2}}{4 \pi}\right) D \partial \Phi_{2}+2 i\left(1-\frac{\beta^{2}}{8 \pi}\right) D \partial \Phi_{1}
$$

We note that the stress-energy tensor is renormalized according to eq.(3.17): in this case one of the fermionic roots has nonvanishing norm. For the spin- $\frac{3}{2}$ current one obtains the following result

$$
\begin{aligned}
W^{\left(\frac{3}{2}\right)}= & -i\left(1-\frac{\beta^{2}}{4 \pi}\right) \partial^{2} \Phi_{2}+i\left(1-\frac{\beta^{2}}{2 \pi}\right) D \Phi_{1} D \partial \Phi_{1}-\left(\partial \Phi_{2}\right)^{2}-i D \Phi_{2} D \partial \Phi_{2} \\
& +2\left(1-\frac{\beta^{2}}{4 \pi}\right) D \Phi_{1} D \partial \Phi_{2}-2 i D \Phi_{1} D \Phi_{2} \partial \Phi_{2}
\end{aligned}
$$

The quantum version for the spin-2 current can be obtained from eqs. (3.27) and (3.28) since $W^{(2)}$ is linearly dependent on $W^{(1)}$ and $W^{\left(\frac{3}{2}\right)}$.

\section{3) A(1,1) Toda theory}


For the rank-2 superalgebra $A(1,1)$ an explicit realization of the fermionic simple roots is (cfr. eq.(A.24))

$$
\begin{aligned}
& \vec{\alpha}_{1}=\vec{\varepsilon}_{1}-\vec{\delta}_{1}=(1,-i) \\
& \vec{\alpha}_{2}=\vec{\varepsilon}_{1}+\vec{\delta}_{1}=(1, i) \\
& \vec{\alpha}_{3}=-\vec{\alpha}_{1}
\end{aligned}
$$

Using this basis, the Toda action takes the form

$$
S=\frac{1}{\beta^{2}} \int d^{2} z d^{2} \theta\left[D \Phi_{1} \bar{D} \Phi_{1}+D \Phi_{2} \bar{D} \Phi_{2}+e^{\Phi_{1}-i \Phi_{2}}+e^{\Phi_{1}+i \Phi_{2}}+e^{-\Phi_{1}+i \Phi_{2}}\right]
$$

The Miura operator in eq.(2.27) for $n=1$ becomes

$$
D\left(D-D \Phi_{1}+i D \Phi_{2}\right)\left(D+2 i D \Phi_{2}\right)\left(D+D \Phi_{1}+i D \Phi_{2}\right)=D^{4}+\sum_{i=0}^{2} W^{\left(\frac{3}{2}-\frac{i}{2}\right)} D^{i}
$$

where the spin- $s$ classical currents are

$$
\begin{aligned}
W^{\left(\frac{1}{2}\right)} & =-2 \partial \Phi_{2}+2 i D \Phi_{2} D \Phi_{1} \\
W^{(1)} & =i D \Phi_{1} \partial \Phi_{1}+i D \Phi_{2} \partial \Phi_{2}-i D \partial \Phi_{1}-D \partial \Phi_{2}+i D\left(D \Phi_{2} D \Phi_{1}\right) \\
W^{\left(\frac{3}{2}\right)} & =-D\left(W^{(1)}-D W^{\left(\frac{1}{2}\right)}\right)
\end{aligned}
$$

The classical stress-energy tensor is given by the holomorphic current $W^{(1)}$, whereas $W^{\left(\frac{1}{2}\right)}$ and $W^{\left(\frac{3}{2}\right)}$ simply satisfy the conservation equations

$$
\bar{D} W^{\left(\frac{1}{2}\right)}=-2 D e^{-\Phi_{1}+i \Phi_{2}} \quad \bar{D} W^{\left(\frac{3}{2}\right)}=-2 i D \partial e^{-\Phi_{1}+i \Phi_{2}}
$$

The expressions in eq.(3.32) give also the correct currents for the quantum system since in this case no anomalies appear in the study of the quantum conservation laws in eq.(3.4).

\section{Supercurrents of affine supersymmetric Toda the- ories}

We consider now the untwisted affine supersymmetric Toda theories described by the action in eq.(2.8). These models are not conformally invariant due to the presence of a mass scale. The conservation equation of the stress-energy tensor is then modified by the appearance of a nonvanishing trace $\bar{T}$

$$
\bar{D} T+D \bar{T}=0
$$


where $T$ can still be written in the form

$$
T=-i D \vec{\Phi} \cdot \partial \vec{\Phi}+2 i \vec{\rho} \cdot D \partial \vec{\Phi}
$$

In order to exhibit the integrability of these theories we have to prove that they possess higher-spin currents $J^{(s)}, s>1$, satisfying the conservation law

$$
\bar{D} J^{(s)}+D \bar{J}^{(s)}=0
$$

which reduces to eq.(4.1) for $s=1$. As for the bosonic case [11], the classical integrability should be guaranteed by the Lax equations (2.9) [16]. However, for affine Toda theories based on Lie superalgebras a general procedure is still lacking.

For the simplest cases of the $D^{(1)}(2,1), B^{(1)}(1,1)$ and $A^{(1)}(1,1)$ theories we have established the existence of higher-spin conserved currents at the classical and quantum level, by using the approach described in the previous section. We compute

$$
\bar{D}_{Z}\left\langle J^{(s)}(Z, \bar{Z})\right\rangle \equiv \bar{D}_{Z}\left\langle J^{(s)}(Z, \bar{Z}) \exp \left(\frac{i}{\beta^{2}} \int d^{2} w d^{2} \theta^{\prime} \mathcal{L}_{\text {int }}\right)\right\rangle_{0}
$$

and search for anomalous local terms which are not expressible as $D$-derivatives of some appropriate $\bar{J}$. Any current of the form $J^{(s)}=D \mathcal{J}^{\left(s-\frac{1}{2}\right)}$ is not relevant since it trivially satisfies eq.(4.3). Furthermore, since we are not planning to determine the actual form of $\bar{J}$, in the course of the calculation we drop total $D$-derivatives and freely integrate by parts on $z, \theta$. We concentrate here on the explicit construction of the quantum $J^{(s)}$ conserved currents up to total $D$-derivatives.

\section{1) $\mathrm{D}^{(1)}(2,1)$ Toda theory}

The untwisted affine extension of the $D(2,1)$ theory is obtained by the addition to the set of simple roots in eq.(3.10) of the corresponding fermionic lowest root $\vec{\alpha}_{0}=$ $-\left(\vec{\alpha}_{1}+\vec{\alpha}_{2}+\vec{\alpha}_{3}\right)=(-1,0,-i)$. The supersymmetric action is

$$
S=\frac{1}{\beta^{2}} \int d^{2} z d^{2} \theta\left[D \vec{\Phi} \cdot \bar{D} \vec{\Phi}+e^{\Phi_{1}-i \Phi_{3}}+e^{-\Phi_{2}+i \Phi_{3}}+e^{\Phi_{2}+i \Phi_{3}}+e^{-\Phi_{1}-i \Phi_{3}}\right]
$$

The theory is still renormalizable; as for the conformal case the only ultraviolet divergences arise from tadpole-type diagrams and they can be cancelled by normal ordering the exponentials in the Lagrangian.

The action in eq.(4.5) is invariant under the following discrete symmetries: $\Phi_{1} \rightarrow-\Phi_{1}$, $\Phi_{2} \rightarrow-\Phi_{2}$ and $\left(\Phi_{1}, \Phi_{2}, \Phi_{3}\right) \rightarrow\left(\Phi_{2}, \Phi_{1},-\Phi_{3}\right)$. Therefore we look for higher-spin currents with a definite parity under these symmetries. An explicit calculation reveals that the theory does not possess conserved currents with spin $s=\frac{3}{2}, 2, \frac{5}{2}$. In particular, the $W^{\left(\frac{3}{2}\right)}$ current present in the conformal case does not survive the affinization since it does not respect the above symmetries. 
The first non trivial conserved current appears at level $s=3$. To find its expression we write the most general linear combination of terms with seven $D$-derivatives acting on the superfields $\Phi_{1}, \Phi_{2}, \Phi_{3}$, even under the symmetries of the Lagrangian. Up to total $D$-derivative contributions, we have

$$
\begin{aligned}
J^{(3)}= & a\left[D \partial \Phi_{1} \partial^{2} \Phi_{1}+D \partial \Phi_{2} \partial^{2} \Phi_{2}\right]+b D \partial \Phi_{3} \partial^{2} \Phi_{3} \\
& +c\left[D \Phi_{1} \partial \Phi_{1} \partial^{2} \Phi_{3}-D \Phi_{2} \partial \Phi_{2} \partial^{2} \Phi_{3}\right]+d\left[\left(\partial \Phi_{1}\right)^{2} D \partial \Phi_{3}-\left(\partial \Phi_{2}\right)^{2} D \partial \Phi_{3}\right] \\
& +e\left[\left(\partial \Phi_{1}\right)^{3} D \Phi_{1}+\left(\partial \Phi_{2}\right)^{3} D \Phi_{2}\right]+f\left(\partial \Phi_{3}\right)^{3} D \Phi_{3} \\
& +g\left[D \Phi_{1} \partial \Phi_{1}\left(\partial \Phi_{3}\right)^{2}+D \Phi_{2} \partial \Phi_{2}\left(\partial \Phi_{3}\right)^{2}\right] \\
& +h\left[D \Phi_{1} D \partial \Phi_{1} D \Phi_{3} \partial \Phi_{3}+D \Phi_{2} D \partial \Phi_{2} D \Phi_{3} \partial \Phi_{3}\right] \\
& +j\left[\left(\partial \Phi_{1}\right)^{2} \partial \Phi_{3} D \Phi_{3}+\left(\partial \Phi_{2}\right)^{2} \partial \Phi_{3} D \Phi_{3}\right] \\
& +(k+i n) D \Phi_{1} \partial \Phi_{1}\left(\partial \Phi_{2}\right)^{2}+n D \Phi_{1} D \partial \Phi_{1} D \Phi_{2} \partial \Phi_{2}+k\left(\partial \Phi_{1}\right)^{2} \partial \Phi_{2} D \Phi_{2} \\
& +q D \Phi_{1} \partial \Phi_{1} D \Phi_{2} \partial \Phi_{2} D \Phi_{3}
\end{aligned}
$$

We insert $J^{(3)}$ in eq.(4.4) and perform the Wick contractions with the interaction Lagrangian. We can exploit the symmetries of the action and the current, so that we need consider only contractions with one exponential. Using identities valid up to integration by parts and dropping total $D$-derivatives, the resulting local contributions can be arranged as follows $\left(\alpha \equiv \frac{\beta^{2}}{2 \pi}\right)$ :

$$
\begin{aligned}
& \bar{D}\left\langle J^{(3)}\left(\frac{i}{\beta^{2}} \int d^{2} w d^{2} \theta^{\prime} e^{\Phi_{1}-i \Phi_{3}}\right)\right\rangle \\
& \sim\left(\left[i c+i d-\frac{\alpha}{2} g-\frac{\alpha}{2} j+\left(1+\frac{\alpha}{2}\right)(k+i n)+\left(2+\frac{\alpha}{2}\right) k+i n\right.\right. \\
& \left.-\left(1+\frac{\alpha}{2}\right) q\right] \partial \Phi_{2} \partial^{2} \Phi_{2} \\
& +\left[-\frac{1}{2} c-\frac{i \alpha}{4} g-\frac{\alpha}{4} h+i\left(1+\frac{\alpha}{4}\right) k-\frac{1}{2} n-\frac{i}{2}\left(1+\frac{\alpha}{2}\right) q\right] D \Phi_{2} D \partial^{2} \Phi_{2} \\
& +\left[i g+i j-i(k+i n)-i k+\frac{i}{2} q\right]\left(\partial \Phi_{2}\right)^{2} \partial \Phi_{3}+\left[-g+i h+k-\frac{1}{2} q\right] D \Phi_{2} D \partial \Phi_{2} \partial \Phi_{3} \\
& +\left[-g+\frac{\alpha}{4} q\right] D \Phi_{2} \partial \Phi_{2} D \partial \Phi_{3}+\left[\frac{i}{2} h+j-(k+i n)+\frac{i}{2} n\right] \partial \Phi_{2} D \partial \Phi_{2} D \Phi_{3} \\
& +\left[-\frac{i}{2} h+\frac{i}{2} n\right] D \Phi_{2} \partial^{2} \Phi_{2} D \Phi_{3}+\left[n-i k+\frac{i}{2}\left(1+\frac{\alpha}{2}\right) q\right] D \Phi_{2} \partial \Phi_{2} D \partial \Phi_{1} \\
& +\left[-a-\frac{i}{2}\left(1+\frac{\alpha}{2}\right) c-i\left(1+\frac{\alpha}{4}\right) d+\left(1+\frac{9 \alpha}{4}+\frac{\alpha^{2}}{4}\right) e\right. \\
& \left.-\left(\frac{\alpha}{4}+\frac{\alpha^{2}}{8}\right) g-\frac{i \alpha}{4} h-\left(\frac{\alpha}{2}+\frac{\alpha^{2}}{8}\right) j\right] \partial \Phi_{1} \partial^{2} \Phi_{1} \\
& +\left[i a-\frac{1}{2}\left(-1+\frac{\alpha}{2}\right) c-\frac{\alpha}{4} d+i\left(2+\frac{3 \alpha}{4}-\frac{\alpha^{2}}{4}\right) e-i\left(\frac{5 \alpha}{4}-\frac{\alpha^{2}}{8}\right) g\right. \\
& \left.+\frac{1}{2}\left(1-\frac{\alpha}{2}\right) h-i\left(1+\alpha-\frac{\alpha^{2}}{8}\right) j\right] \partial \Phi_{3} \partial^{2} \Phi_{1}
\end{aligned}
$$




$$
\begin{aligned}
& +\left[i b-\left(1+\frac{\alpha}{4}\right) c-\left(1+\frac{\alpha}{4}\right) d+2 i e+i\left(\frac{3 \alpha}{4}+\frac{\alpha^{2}}{4}\right) f-i\left(1+\alpha+\frac{\alpha^{2}}{8}\right) g\right. \\
& \left.+\frac{1}{2} h-i\left(2+\frac{5 \alpha}{4}+\frac{\alpha^{2}}{8}\right) j\right] \partial \Phi_{1} \partial^{2} \Phi_{3} \\
& +\left[b+\frac{i \alpha}{4} c+\frac{i \alpha}{4} d+\left(1-\frac{9 \alpha}{4}+\frac{\alpha^{2}}{4}\right) f+\left(\frac{\alpha}{2}-\frac{\alpha^{2}}{8}\right) g+\left(\frac{\alpha}{4}-\frac{\alpha^{2}}{8}\right) j\right] \partial \Phi_{3} \partial^{2} \Phi_{3} \\
& +\left[-\frac{i}{2} c-\frac{3 \alpha}{4} f+\left(1+\frac{\alpha}{2}\right) g+\frac{i}{2}\left(1-\frac{\alpha}{4}\right) h+\left(1+\frac{\alpha}{4}\right) j\right] D \Phi_{1} D \partial^{2} \Phi_{3} \\
& +\left[\frac{i}{2} c-\left(3+\frac{3 \alpha}{4}\right) e+\frac{\alpha}{4} g+\frac{i \alpha}{8} h+\left(1+\frac{\alpha}{2}\right) j\right] D \Phi_{3} D \partial^{2} \Phi_{1} \\
& +\left[i d+3 e+\frac{\alpha}{2} g+\frac{i}{2}(1-\alpha) h-\left(1+\frac{\alpha}{2}\right) j\right] D \partial \Phi_{1} D \partial \Phi_{3} \\
& +\left[-3 i e+i g-\frac{1}{2} h+2 i j\right] D \partial \Phi_{1} D \Phi_{3} \partial \Phi_{3}+[2 e+2 f-2 g-2 j]\left(\partial \Phi_{3}\right)^{2} \partial \Phi_{1} \\
& \left.+\left[-3 i f+2 i g+\frac{1}{2} h+i j\right] \partial \Phi_{3} D \partial \Phi_{3} D \Phi_{1}\right) e^{\Phi_{1}-i \Phi_{3}}
\end{aligned}
$$

Since the various terms in the r.h.s. of eq.(4.7) are all independent up to total $D^{-}$ derivatives, the $J^{(3)}$ current is conserved only if the coefficients separately vanish. This condition gives a system of equations which determine the unknowns $a, b, \cdots, q$ uniquely, up to an overall factor. The $J^{(3)}$ quantum conserved current is

$$
\begin{aligned}
J^{(3)=} & -i\left(4+\frac{3 \beta^{2}}{8 \pi}-\frac{35 \beta^{4}}{64 \pi^{2}}\right)\left[D \partial \Phi_{1} \partial^{2} \Phi_{1}+D \partial \Phi_{2} \partial^{2} \Phi_{2}\right] \\
& -i\left(1-\frac{3 \beta^{2}}{4 \pi}-\frac{35 \beta^{4}}{64 \pi^{2}}\right) D \partial \Phi_{3} \partial^{2} \Phi_{3}+i\left(1+\frac{3 \beta^{2}}{8 \pi}\right)\left(\partial \Phi_{3}\right)^{3} D \Phi_{3} \\
& -6\left(1+\frac{3 \beta^{2}}{8 \pi}\right)\left[D \Phi_{1} \partial \Phi_{1} \partial^{2} \Phi_{3}-D \Phi_{2} \partial \Phi_{2} \partial^{2} \Phi_{3}\right] \\
& +6\left(1+\frac{\beta^{2}}{4 \pi}\right)\left[\left(\partial \Phi_{1}\right)^{2} D \partial \Phi_{3}-\left(\partial \Phi_{2}\right)^{2} D \partial \Phi_{3}\right] \\
& -i\left(1-\frac{3 \beta^{2}}{8 \pi}\right)\left[\left(\partial \Phi_{1}\right)^{3} D \Phi_{1}+\left(\partial \Phi_{2}\right)^{3} D \Phi_{2}\right] \\
& -i \frac{3 \beta^{2}}{2 \pi}\left[D \Phi_{1} \partial \Phi_{1}\left(\partial \Phi_{3}\right)^{2}+D \Phi_{2} \partial \Phi_{2}\left(\partial \Phi_{3}\right)^{2}\right] \\
& -6\left(1+\frac{5 \beta^{2}}{8 \pi}\right)\left[D \Phi_{1} D \partial \Phi_{1} D \Phi_{3} \partial \Phi_{3}+D \Phi_{2} D \partial \Phi_{2} D \Phi_{3} \partial \Phi_{3}\right] \\
& +i \frac{9 \beta^{2}}{4 \pi}\left[\left(\partial \Phi_{1}\right)^{2} \partial \Phi_{3} D \Phi_{3}+\left(\partial \Phi_{2}\right)^{2} \partial \Phi_{3} D \Phi_{3}\right] \\
& -6 i\left(1+\frac{\beta^{2}}{4 \pi}\right) D \Phi_{1} \partial \Phi_{1}\left(\partial \Phi_{2}\right)^{2}-6\left(1+\frac{5 \beta^{2}}{8 \pi}\right) D \Phi_{1} D \partial \Phi_{1} D \Phi_{2} \partial \Phi_{2} \\
& +i \frac{9 \beta^{2}}{4 \pi}\left(\partial \Phi_{1}\right)^{2} \partial \Phi_{2} D \Phi_{2}-12 i D \Phi_{1} \partial \Phi_{1} D \Phi_{2} \partial \Phi_{2} D \Phi_{3}
\end{aligned}
$$

We note that the classical current obtained in the limit $\beta^{2} \rightarrow 0$ can be written, up to 
total $D$-derivative terms, as a function of the $W$ and $F^{(1)}$ currents in eqs. 3.14, 3.15)

$$
J^{(3)}=W^{(1)} D W^{(1)}+2 W^{(1)} W^{\left(\frac{3}{2}\right)}+4 F^{(1)} D F^{(1)}
$$

In the conformal case the three terms are separately holomorphic currents, whereas in the perturbed theory only the linear combination in eq.(4.9) satisfies the classical conservation law. At the quantum level, since $J^{(3)}$ is a composite operator we could not simply insert the quantum expressions of the $W^{(1)}$ and $W^{\left(\frac{3}{2}\right)}$ currents in eq.(4.9). The lengthy calculation in eq. (4.7) was needed in order to obtain the complete renormalized form of $J^{(3)}$

\section{2) $\mathbf{B}^{(1)}(\mathbf{1}, 1)$ Toda theory}

With the choice of simple roots in eq.(3.22), the lowest root of the $B^{(1)}(1,1)$ model is given by $\vec{\alpha}_{0}=-\left(\vec{\alpha}_{1}+2 \vec{\alpha}_{2}\right)=(-1,-i)$. The affine action is

$$
S=\frac{1}{\beta^{2}} \int d^{2} z d^{2} \theta\left[D \vec{\Phi} \cdot \bar{D} \vec{\Phi}+e^{\Phi_{1}-i \Phi_{2}}+2 e^{i \Phi_{2}}+e^{-\Phi_{1}-i \Phi_{2}}\right]
$$

This system does not have nontrivial conserved currents of $\operatorname{spin} s=\frac{3}{2}$ or $s=2$. In particular the $W^{\left(\frac{3}{2}\right)}$ current is not conserved in the affine case because it does not respect the discrete symmetry $\Phi_{1} \rightarrow-\Phi_{1}$ of the action.

The first higher-spin current appears at $s=3$. Its construction is performed following the same steps as for the $D^{(1)}(2,1)$ case. We consider up to total $D$-derivative terms the most general expression, even in $\Phi_{1}$

$$
\begin{aligned}
J^{(3)} & =a D \partial \Phi_{1} \partial^{2} \Phi_{1}+b D \partial \Phi_{2} \partial^{2} \Phi_{2}+c D \Phi_{1} \partial \Phi_{1} \partial^{2} \Phi_{2}+d\left(\partial \Phi_{1}\right)^{2} D \partial \Phi_{2} \\
& +e\left(\partial \Phi_{1}\right)^{3} D \Phi_{1}+f\left(\partial \Phi_{2}\right)^{3} D \Phi_{2}+g D \Phi_{1} \partial \Phi_{1}\left(\partial \Phi_{2}\right)^{2} \\
& +h D \Phi_{1} D \partial \Phi_{1} D \Phi_{2} \partial \Phi_{2}+k\left(\partial \Phi_{1}\right)^{2} \partial \Phi_{2} D \Phi_{2}
\end{aligned}
$$

and compute all the local contributions which arise from Wick contracting with the interaction Lagrangian. The anomaly cancellation requirement leads to a set of equations for $a, b, \ldots, k$ which can be solved nontrivially. Details of the calculation are contained in Ref.[15]. We quote here the result for the quantum $J^{(3)}$ current

$$
\begin{aligned}
J^{(3)} & =i\left(-4+\frac{13}{8 \pi} \beta^{2}+\frac{7}{32 \pi^{2}} \beta^{4}-\frac{\beta^{6}}{64 \pi^{3}}\right) D \partial \Phi_{1} \partial^{2} \Phi_{1} \\
& -i\left(1-\frac{7}{8 \pi} \beta^{2}-\frac{7}{32 \pi^{2}} \beta^{4}+\frac{\beta^{6}}{64 \pi^{3}}\right) D \partial \Phi_{2} \partial^{2} \Phi_{2} \\
& +\left(-6+\frac{3}{2 \pi} \beta^{2}\right) D \Phi_{1} \partial \Phi_{1} \partial^{2} \Phi_{2}+\left(6-\frac{9}{4 \pi} \beta^{2}+\frac{3}{16 \pi^{2}} \beta^{4}\right)\left(\partial \Phi_{1}\right)^{2} D \partial \Phi_{2} \\
& -i\left(1-\frac{\beta^{2}}{2 \pi}\right)\left(\partial \Phi_{1}\right)^{3} D \Phi_{1}+i\left(1+\frac{\beta^{2}}{4 \pi}\right)\left(\partial \Phi_{2}\right)^{3} D \Phi_{2} \\
& -6 D \Phi_{1} D \partial \Phi_{1} D \Phi_{2} \partial \Phi_{2}+\frac{3 i}{4 \pi} \beta^{2}\left(\partial \Phi_{1}\right)^{2} \partial \Phi_{2} D \Phi_{2}
\end{aligned}
$$


The classical current is obtained by setting $\beta^{2}=0$. At the classical level $J^{(3)}$ is equivalent, up to total $D$-derivative terms, to the classical current $W^{(1)} D W^{(1)}+2 W^{(1)} W^{\left(\frac{3}{2}\right)}$.

\section{3) $\mathrm{A}^{(1)}(1,1)$ Toda theory}

A particular realization of the simple roots for the $A(1,1)$ superalgebra is the one in eq. (3.29). The corresponding fermionic lowest root is then given by $\vec{\alpha}_{0}=-\vec{\alpha}_{2}=(-1,-i)$. The $A^{(1)}(1,1)$ affine Toda action is

$$
S=\frac{1}{\beta^{2}} \int d^{2} z d^{2} \theta\left[D \vec{\Phi} \cdot \bar{D} \vec{\Phi}+e^{\Phi_{1}-i \Phi_{2}}+e^{\Phi_{1}+i \Phi_{2}}+e^{-\Phi_{1}+i \Phi_{2}}+e^{-\Phi_{1}-i \Phi_{2}}\right]
$$

It is symmetric under $\Phi_{1} \rightarrow-\Phi_{1}$ and $\Phi_{2} \rightarrow-\Phi_{2}$. The first nontrivial conserved current appears again at $s=3$. The most general linear combination of terms with seven $D$ derivatives, even under the above mentioned discrete symmetries is

$$
\begin{aligned}
J^{(3)=} & a D \partial \Phi_{1} \partial^{2} \Phi_{1}+b D \partial \Phi_{2} \partial^{2} \Phi_{2}+c D \Phi_{1}\left(\partial \Phi_{1}\right)^{3}+d D \Phi_{2}\left(\partial \Phi_{2}\right)^{3} \\
& +e\left(\partial \Phi_{1}\right)^{2} D \Phi_{2} \partial \Phi_{2}+f\left(\partial \Phi_{2}\right)^{2} D \Phi_{1} \partial \Phi_{1} \\
& +g D \Phi_{1} D \partial \Phi_{1} D \Phi_{2} \partial \Phi_{2}
\end{aligned}
$$

We find $\left(\alpha \equiv \frac{\beta^{2}}{2 \pi}\right)$

$$
\begin{aligned}
& \bar{D}\left\langle J^{(3)}\left(\frac{i}{\beta^{2}} \int d^{2} w d^{2} \theta^{\prime} e^{\Phi_{1}+i \Phi_{2}}\right)\right\rangle \\
& \sim\left(\left[-a+\left(1+\frac{9 \alpha}{4}+\frac{\alpha^{2}}{4}\right) c-\left(\frac{\alpha}{2}+\frac{\alpha^{2}}{8}\right) e-\left(\frac{\alpha}{4}+\frac{\alpha^{2}}{8}\right) f-\frac{i \alpha}{4} g\right] \partial \Phi_{1} \partial^{2} \Phi_{1}\right. \\
& +\left[b+\left(1-\frac{9 \alpha}{4}+\frac{\alpha^{2}}{4}\right) d+\left(\frac{\alpha}{4}-\frac{\alpha^{2}}{8}\right) e+\left(\frac{\alpha}{2}-\frac{\alpha^{2}}{8}\right) f\right] \partial \Phi_{2} \partial^{2} \Phi_{2} \\
& +\left[-i a-i\left(2+\frac{3 \alpha}{4}-\frac{\alpha^{2}}{4}\right) c+i\left(1+\alpha-\frac{\alpha^{2}}{8}\right) e+i\left(\frac{5 \alpha}{4}-\frac{\alpha^{2}}{8}\right) f\right. \\
& +\left[-i b-2 i c-i\left(\frac{3 \alpha}{4}+\frac{\alpha^{2}}{4}\right) d+i\left(2+\frac{5 \alpha}{4}+\frac{\alpha^{2}}{8}\right) e+i\left(1+\alpha+\frac{\alpha^{2}}{8}\right) f-\frac{1}{2} g\right] \partial \Phi_{1} \partial^{2} \Phi_{2} \\
& +\left[-3 c+\left(1+\frac{\alpha}{2}\right) e-\frac{\alpha}{2} f-\frac{i}{2}(1-\alpha) g\right] D \partial \Phi_{1} D \partial \Phi_{2} \\
& +\left[-3\left(1+\frac{\alpha}{4}\right) c+\left(1+\frac{\alpha}{2}\right) e+\frac{\alpha}{4} f+\frac{i \alpha}{8} g\right] D \partial^{2} \Phi_{1} D \Phi_{2} \\
& +\left[\frac{3 \alpha}{4} d-\left(1+\frac{\alpha}{4}\right) e-\left(1+\frac{\alpha}{2}\right) f+\frac{i}{2}\left(-1+\frac{\alpha}{4}\right) g\right] D \Phi_{1} D \partial^{2} \Phi_{2} \\
& +\left[3 i d-i e-2 i f-\frac{1}{2} g\right] D \Phi_{1} \partial \Phi_{2} D \partial \Phi_{2}+[2 c+2 d-2 e-2 f] \partial \Phi_{1}\left(\partial \Phi_{2}\right)^{2} \\
& \left.+\left[-3 i c+2 i e+i f-\frac{1}{2} g\right] D \partial \Phi_{1} D \Phi_{2} \partial \Phi_{2}\right) e^{\Phi_{1}+i \Phi_{2}} \\
& +\left[-\left(-\frac{\alpha}{2}\right)\right.
\end{aligned}
$$


where the result has been expressed in terms of an independent set. In this case too the contractions with the other exponentials need not be considered on the basis of the symmetries of the Lagrangian. The quantum current is determined, up to an overall normalization factor, by imposing that the r.h.s. vanishes. We find

$$
\begin{aligned}
J^{(3)}= & -i\left(1-\frac{11 \beta^{4}}{16 \pi^{2}}\right)\left[D \partial \Phi_{1} \partial^{2} \Phi_{1}+D \partial \Phi_{2} \partial^{2} \Phi_{2}\right]-i\left(1-\frac{3 \beta^{2}}{4 \pi}\right) D \Phi_{1}\left(\partial \Phi_{1}\right)^{3} \\
& +i\left(1+\frac{3 \beta^{2}}{4 \pi}\right) D \Phi_{2}\left(\partial \Phi_{2}\right)^{3}-i\left(3-\frac{9 \beta^{2}}{4 \pi}\right)\left(\partial \Phi_{1}\right)^{2} D \Phi_{2} \partial \Phi_{2} \\
& +i\left(3-\frac{3 \beta^{2}}{4 \pi}\right)\left(\partial \Phi_{2}\right)^{2} D \Phi_{1} \partial \Phi_{1}-\frac{3 \beta^{2}}{\pi} D \Phi_{1} D \partial \Phi_{1} D \Phi_{2} \partial \Phi_{2}
\end{aligned}
$$

Setting $\beta^{2}=0$ we obtain the classical $J^{(3)}$ current which is expressible as a linear combination of the $W$-currents

$$
J^{(3)}=W^{(1)} D W^{(1)}-i W^{(1)} \partial W^{\left(\frac{1}{2}\right)}+\frac{1}{2}\left(W^{\left(\frac{1}{2}\right)}\right)^{2} W^{(1)}
$$

\section{The mass spectrum}

In this section we study the classical mass spectrum of the fundamental particles of the affine Toda theories based on the $D^{(1)}(n+1, n), B^{(1)}(n, n)$ and $A^{(1)}(n, n)$ superalgebras, and for the first simplest cases we compute one-loop corrections to the masses. From the general expression of the affine supersymmetric Toda action in eq.(2.8), expanding the potential up to third order, we obtain the mass matrix and the three-point couplings

$$
\begin{aligned}
S & =\frac{1}{\beta^{2}} \int d^{2} z d^{2} \theta\left[D \vec{\Phi} \cdot \bar{D} \vec{\Phi}+\frac{1}{2} \sum_{i=0}^{r} q_{i} \alpha_{i}^{a} \alpha_{i}^{b} \Phi_{a} \Phi_{b}+\frac{1}{3 !} \sum_{i=0}^{r} q_{i} \alpha_{i}^{a} \alpha_{i}^{b} \alpha_{i}^{c} \Phi_{a} \Phi_{b} \Phi_{c}+O\left(\Phi^{4}\right)\right] \\
& \equiv \frac{1}{\beta^{2}} \int d^{2} z d^{2} \theta\left[\vec{\Phi}(\bar{D} D-M) \vec{\Phi}+c^{a b c} \Phi_{a} \Phi_{b} \Phi_{c}+O\left(\Phi^{4}\right)\right]
\end{aligned}
$$

The first order term is zero due to the definition of the Kač labels $q_{i}\left(\sum_{i=0}^{r} q_{i} \overrightarrow{\alpha_{i}}=0\right)$. The second order term contains the matrix

$$
M^{a b} \equiv-\frac{1}{2} \sum_{i=0}^{r} q_{i} \alpha_{i}^{a} \alpha_{i}^{b}
$$

whose eigenvalues give the supersymmetric classical mass spectrum. In terms of the mass eigenvalues $M_{k}$, the masses of the bosonic particles are $m_{k}^{2}=2 M_{k}^{2}$. With the choice of simple roots listed in the Appendix, the mass matrix in eq.(5.2) for the $D^{(1)}(n+1, n)$, $B^{(1)}(n, n)$ and $A^{(1)}(n, n)$ models is not diagonal, so that in order to determine the mass spectrum one has to solve the corresponding eigenvalue problem. We have not succeeded in doing this for general $n$, but we have studied a number of theories and guessed the 
general expression of the masses $m_{k}$. They are given by

$$
\begin{aligned}
D^{(1)}(n+1, n): & & k=1, \cdots, 2 n-1 \\
& m_{k}^{2}=8 \sin ^{2} \frac{2 \pi k}{h} & \\
B^{(1)}(n, n): & : & k=1, \cdots, 2 n-1 \\
& m_{k}^{2}=8 \sin ^{2} \frac{2 \pi k}{h} & \\
& m_{2 n}^{2}=2 & \\
A^{(1)}(n, n): & m_{k}^{2}=m_{2 n+1-k}^{2}=8 \sin ^{2} \frac{2 \pi k}{h} & k=1, \cdots, n
\end{aligned}
$$

where $h$ is the Coxeter number of the superalgebras, $h=4 n$ for $D^{(1)}(n+1, n)$ and $B^{(1)}(n, n), h=2 n+2$ for $A^{(1)}(n, n)$. We have checked our guess with the computer program Mathematica for the cases $n=1, \cdots, 6$.

We note that the mass spectrum for the $B^{(1)}(n, n)$ theory is naturally obtained from the spectrum of the $D^{(1)}(n+1, n)$ model by setting $\Phi_{2 n+1} \equiv 0$, which corresponds to the folding operation from the $B^{(1)}(n, n)$ Dynkin diagram to the $D^{(1)}(n+1, n)$ one.

We stress that the masses of all these theories are real despite the manifest nonhermiticity of the action in eq.(2.8).

In the $D$-theories the $n, 2 n$ and $2 n+1$ particles are at threshold, due to the particular mass relations $m_{n}=2 m_{2 n}=2 m_{2 n+1}$. In the same way we have $m_{n}=2 m_{2 n}$ for the $B$ theories. As a result of this, divergent contributions similar to the ones discussed in Ref. [17], could be produced in on-shell amplitudes. However, one can show that wavefunction renormalizations are sufficient to make these theories well defined.

In order to compute one-loop mass corrections one needs evaluate on-shell self-energy supergraphs. It is convenient to perform the calculation using a basis of superfields in which the mass matrix $M$ is diagonal. In this way we have massive propagators for each internal line of a Feynman supergraph

$$
\left\langle\Phi_{i}(Z, \bar{Z}) \Phi_{j}(0,0)\right\rangle=-i \delta_{i j} \beta^{2} \frac{\left(\bar{D} D+M_{i}\right)}{\square+2 M_{i}^{2}} \delta^{(2)}(\theta)
$$

whereas external lines satisfy the on-shell condition $\bar{D} D \Phi_{j}=M_{j} \Phi_{j}$. The one-loop contribution to the effective action $\left(e^{i S} \rightarrow e^{i \Gamma}\right)$ from a generic self-energy diagram with particle $i$ on the external lines, and $j, k$ internal, is of the form

$$
\int d^{2} \theta \Phi_{i}\left(\bar{D} D+M_{j}+M_{k}\right) \Phi_{i} \Sigma\left(p_{i}^{2} ; m_{j}^{2}, m_{k}^{2}\right)
$$

where

$$
\Sigma\left(p_{i}^{2} ; m_{j}^{2}, m_{k}^{2}\right)=\frac{1}{(2 \pi)^{2}} \int \frac{d^{2} k}{\left(k^{2}-m_{j}^{2}\right)\left[\left(k-p_{i}\right)^{2}-m_{k}^{2}\right]}
$$


We have computed one-loop mass corrections for the $D^{(1)}(2,1)$ and $D^{(1)}(3,2)$ theories, for the $B^{(1)}(1,1)$ and $B^{(1)}(2,2)$ theories and finally for the $A^{(1)}(1,1)$ and $A^{(1)}(2,2)$ models. As already mentioned, in the $D$ and $B$ cases threshold divergences are produced at intermediate stages of the calculation but they all cancel in the final answer. We have found that in the $D^{(1)}(n+1, n)$ and $A^{(1)}(n, n)$ theories with $n=1,2$, the classical mass spectrum is stable under quantization, since the couplings and the masses for these theories are such that the on-shell contributions from self-energy supergraphs always sum up to zero. Absence of one-loop mass renormalization is verified also for the two supermultiplets described by the $B^{(1)}(1,1)$ model. At level $n=2$, while the masses of the two superfields at threshold, i.e. the lightest and the heaviest, are still not renormalized, nonvanishing mass contributions are produced for the the other two. In general we expect that the classical mass ratios of the $B^{(1)}(n, n)$ models will be spoiled by quantum corrections following a pattern which is typical of bosonic nonsimply-laced Toda theories. We show this explicitly on the $B^{(1)}(2,2)$ example.

The classical Lagrangian of the $B^{(1)}(2,2)$ theory is given by

$$
\mathcal{L}=D \vec{\Phi} \cdot \bar{D} \vec{\Phi}+e^{\Phi_{1}-i \Phi_{3}}+2 e^{i \Phi_{3}-\Phi_{2}}+2 e^{\Phi_{2}-i \Phi_{4}}+2 e^{i \Phi_{4}}+e^{-\Phi_{1}-i \Phi_{3}}
$$

In this basis, which corresponds to the realization of roots as in eq.(A.19,A.20) for $n=2$, the mass matrix is not diagonal. We obtain a diagonal basis through the change of variables

$$
\begin{aligned}
& \Phi_{1} \rightarrow \Phi_{4} \\
& \Phi_{3} \rightarrow \frac{\sqrt{\sqrt{2}+1}}{2} \Phi_{1}+\frac{i \sqrt{\sqrt{2}-1}}{\sqrt{2}} \Phi_{1}+\frac{\sqrt{\sqrt{2}+1}}{\sqrt{2}} \Phi_{3}-\frac{i \sqrt{\sqrt{2}-1}}{2} \Phi_{3} \\
& \Phi_{2} \rightarrow \frac{\sqrt{\sqrt{2}+1}}{2} \Phi_{1}-\frac{1}{\sqrt{2}} \Phi_{2}-\frac{i \sqrt{\sqrt{2}-1}}{2} \Phi_{3}
\end{aligned}
$$

The relabeling of the fields has been chosen in such a way that the corresponding masses are the ones in eq.(5.4); specifically we have

$$
m_{1}^{2}=4 \quad m_{2}^{2}=8 \quad m_{3}^{2}=4 \quad m_{4}^{2}=2
$$

The three-point couplings in the new basis are

$$
\begin{aligned}
\mathcal{L}^{(3)}= & \frac{i}{\sqrt{2}} \Phi_{2} \Phi_{1}^{2}+\frac{i}{\sqrt{2}} \Phi_{2} \Phi_{3}^{2}+\sqrt{2} \Phi_{1} \Phi_{2} \Phi_{3} \\
& -\frac{i}{\sqrt{2}} \Phi_{2} \Phi_{4}^{2}-\frac{i \sqrt{\sqrt{2}+1}}{2} \Phi_{1} \Phi_{4}^{2}-\frac{\sqrt{\sqrt{2}-1}}{2} \Phi_{3} \Phi_{4}^{2}
\end{aligned}
$$

One-loop mass corrections arise from the diagrams shown in Fig.2. 
In terms of the momentum integrals $\Sigma\left(p_{i}^{2} ; m_{j}^{2}, m_{k}^{2}\right)$ in eq.(5.8), the contributions from the various supergraphs evaluated on-shell are
(a) : $\frac{1}{\sqrt{2}} \Sigma\left(m_{1}^{2} ; m_{4}^{2}, m_{4}^{2}\right)$
(b) : $\quad-4(1+\sqrt{2}) \Sigma\left(m_{1}^{2} ; m_{1}^{2}, m_{2}^{2}\right)$
(c) : $4 \Sigma\left(m_{1}^{2} ; m_{2}^{2}, m_{3}^{2}\right)$
$(d): \quad-2(\sqrt{2}+1) \Sigma\left(m_{2}^{2} ; m_{1}^{2}, m_{1}^{2}\right)$
(e) : $2(\sqrt{2}-1) \Sigma\left(m_{2}^{2} ; m_{3}^{2}, m_{3}^{2}\right)$
$(f): 0$
$(g): 4 \Sigma\left(m_{2}^{2} ; m_{1}^{2}, m_{3}^{2}\right)$
(h): $4(\sqrt{2}-1) \Sigma\left(m_{3}^{2} ; m_{2}^{2}, m_{3}^{2}\right)$
$(i): \quad-\frac{1}{\sqrt{2}} \Sigma\left(m_{3}^{2} ; m_{4}^{2}, m_{4}^{2}\right)$
(l): $4 \Sigma\left(m_{3}^{2} ; m_{1}^{2}, m_{2}^{2}\right)$
$(m): 0$
$(n): \sqrt{2} \Sigma\left(m_{4}^{2} ; m_{1}^{2}, m_{4}^{2}\right)$
$(o):-\sqrt{2} \Sigma\left(m_{4}^{2} ; m_{3}^{2}, m_{4}^{2}\right)$

Using the equalities $\Sigma\left(m_{1}^{2} ; m_{1}^{2}, m_{2}^{2}\right)=\Sigma\left(m_{1}^{2} ; m_{2}^{2}, m_{3}^{2}\right)$ and $\Sigma\left(m_{1}^{2} ; m_{4}^{2}, m_{4}^{2}\right)=4 \Sigma\left(m_{1}^{2} ; m_{1}^{2}, m_{2}^{2}\right)$ we obtain the one-loop mass corrections

$$
\begin{aligned}
& \Delta M_{1}=-\Delta M_{3}=-i \sqrt{2} \beta^{2} \Sigma\left(m_{1}^{2} ; m_{1}^{2}, m_{2}^{2}\right)=\frac{\beta^{2}}{32 \sqrt{2}} \\
& \Delta M_{2}=\Delta M_{4}=0
\end{aligned}
$$

Therefore the classical mass ratios of the $B^{(1)}(2,2)$ theory are not maintained.

The analysis of the mass spectrum we have presented is not complete since the affine supersymmetric Toda theories admit soliton configurations. At the classical level the presence of such solutions is signalled by the periodicity of the potential under a shift of the superfields associated to the imaginary components of the roots

$$
\vec{\Phi} \rightarrow \vec{\Phi}+2 \pi \vec{\omega}
$$

with $\vec{\omega} \cdot \vec{\alpha}_{i}=k$ and $k$ any integer. For the $D^{(1)}(n+1, n)$ and $B^{(1)}(n, n)$ theories supersymmetric soliton solutions can be constructed in a straightforward manner since setting to zero the superfields associated to the real components of the roots amounts to consider $n$ decoupled supersine-Gordon systems. In these cases one can then borrow all the results in the literature [18] and obtain the complete solitonic spectrum. 
For the $A^{(1)}(n, n)$ theories, when $n \geq 2$ the situation is more complicated: looking at the explicit expressions of the roots in eq.(A.24,A.25) it is clear that the interaction between the $n$ superfields associated to the imaginary components of the roots is highly nontrivial. The general construction of soliton solutions is beyond the scope of this paper.

\section{Conclusions}

We have considered Toda theories based on Lie superalgebras for which an extended set of fermionic roots exist: the corresponding field theories are supersymmetric. More precisely we have given an explicit lagrangian realization in $N=1$ superspace for the $D(n+1, n), B(n, n)$ and $A(n, n)$ theories. These systems, although nonunitary, are quantum integrable. We have constructed the superspace Miura operators and computed the exact renormalized expressions of the currents which determine the W-symmetries of the conformal theories. The existence of quantum conserved higher-spin currents has then been established for specific examples in the untwisted affine cases, namely we have proven conservation to all-loop order of the spin-3 current for the $D^{(1)}(2,1), B^{(1)}(1,1)$ and $A^{(1)}(1,1)$ theories.

The affine theories are massive systems: we have computed one-loop corrections to the particle mass spectrum and found results that parallel the situation for bosonic Toda theories based on simply-laced and nonsimply-laced Lie algebras.

All the calculations we have presented have been obtained taking advantage of a full

superspace approach. We emphasize that this formalism is particularly advantageous in the study of the renormalization of the supercurrents, since a component calculation would have been prohibitively complicated.

Having exhibited the existence of higher-spin charges which commute with the supersymmetric hamiltonians, we could proceed to the determination of the S matrices, which are now guaranteed to be elastic and factorizable. For supersymmetric Toda theories, as compared to unitary Toda systems, things are not so straightforward: the presence of solitonic configurations alters the mass spectrum in a substantial way and the full quantum group approach is then required for the construction of the complete S-matrix |19. 


\section{A Appendix}

In this appendix we list some of the basic properties, the Dynkin diagrams and the set of fermionic simple roots for superalgebras 円 which admit a fermionic untwisted affine extension. We also give the generators and the weights in the fundamental representation, which are needed to construct the Miura transformation for the corresponding Toda theories.

We remind that, given a rank- $r$ superalgebra with a completely fermionic set of simple roots, its generators $h_{a}, e_{i}^{+}, e_{j}^{-}$satisfy

$$
\left[h_{a}, h_{b}\right]=0 \quad\left[h_{a}, e_{j}^{ \pm}\right]= \pm \alpha_{j}^{a} e_{j}^{ \pm} \quad\left\{e_{i}^{+}, e_{j}^{-}\right\}=\delta_{i j} h_{a} \alpha_{i}^{a}
$$

where $\{$,$\} indicates the anticommutation operator. The simple roots \alpha_{j}$ are linear functionals on the Cartan subalgebra $\mathcal{H}$ and they can be realized explicitly as $r$-dimensional vectors with components $\alpha_{j}^{a} \equiv \alpha_{j}\left(h_{a}\right)$. It is convenient to represent the roots in terms of vectors $\vec{\varepsilon}_{i}, \vec{\delta}_{j}$, defined by

$$
\vec{\varepsilon}_{i} \cdot \vec{\varepsilon}_{j}=\delta_{i j} \quad \vec{\delta}_{i} \cdot \vec{\delta}_{j}=-\delta_{i j} \quad \vec{\varepsilon}_{i} \cdot \vec{\delta}_{j}=0
$$

We will give explicit expressions of the generators of the various superalgebras in the fundamental representation. To this end we introduce a set of complex matrices $\mathcal{M}(p \mid q ; \mathcal{C})$ of the form

$$
M=\left(\begin{array}{ll}
A & B \\
C & D
\end{array}\right)
$$

where the dimensions of the submatrices $A, B, C$ and $D$ are respectively $p \times p, p \times q$ $q \times p$ and $q \times q$. The matrix $M$ is said to be even if $C=B=0$, odd if $A=D=0$. The generators $\vec{h}, e_{j}^{ \pm}$are then expressed as $d \times d$ such matrices, being $d=p+q$ the dimension of the fundamental representation of the superalgebra, in terms of the basis

$$
\left(e_{i, j}\right)_{k l}=\delta_{i k} \delta_{j l}
$$

The weights $\lambda$ can be obtained through the following general construction. Given a representation $\Gamma$ for the superalgebra, the weights $\lambda$ are defined by the relation

$$
\Gamma(h) \psi(\lambda)=\lambda(h) \psi(\lambda)
$$

where $\Gamma(h)$ is a diagonal matrix which realizes the $\Gamma$-representation of an element $h$ in the Cartan subalgebra and $\psi(\lambda)$ is a set of common eigenvectors for the matrices $\Gamma(h)$. Since the weights are linear functionals on $\mathcal{H}$, they can be expressed as linear combinations of the simple roots

$$
\lambda=\sum_{j=1}^{r} \mu_{j} \alpha_{j}
$$

\footnotetext{
${ }^{1} \mathrm{~A}$ comprehensive introduction to superalgebras is contained in Ref. 20]
} 
where the set of coefficients $\mu_{j}$ can be determined by solving the system obtained from the relation (A.6) evaluated on each element of the $\mathcal{H}$ basis:

$$
\begin{aligned}
\lambda\left(h_{1}\right) & =\sum_{j=1}^{r} \mu_{j} \alpha_{j}\left(h_{1}\right) \\
\lambda\left(h_{2}\right) & =\sum_{j=1}^{r} \mu_{j} \alpha_{j}\left(h_{2}\right) \\
& \ldots \\
\lambda\left(h_{r}\right) & =\sum_{j=1}^{r} \mu_{j} \alpha_{j}\left(h_{r}\right)
\end{aligned}
$$

Remembering that $\alpha_{j}\left(h_{a}\right)=\alpha_{j}^{a}$ are the components of the simple roots, we can express the coefficients $\mu_{j}$ as linear functions of $\lambda\left(h_{1}\right) \cdots \lambda\left(h_{r}\right)$. Finally, if we define $\lambda_{j}(h) \equiv(\Gamma(h))_{j j}$, the weights in eq.(A.6) are given by

$$
\begin{aligned}
\lambda_{1} & =\sum_{j=1}^{r} \mu_{j}\left(\Gamma\left(h_{1}\right)_{11} \cdots \Gamma\left(h_{r}\right)_{11}\right) \alpha_{j} \\
\lambda_{2} & =\sum_{j=1}^{r} \mu_{j}\left(\Gamma\left(h_{1}\right)_{22} \cdots \Gamma\left(h_{r}\right)_{22}\right) \alpha_{j} \\
& \cdots \\
\lambda_{s} & =\sum_{j=1}^{r} \mu_{j}\left(\Gamma\left(h_{1}\right)_{s s} \cdots \Gamma\left(h_{r}\right)_{s s}\right) \alpha_{j}
\end{aligned}
$$

where $s$ is the dimension of the $\Gamma$-representation. In the following we will work in the fundamental representation.

We explicitly give results for the superalgebras $D(n+1, n), B(n, n)$ and $A(n, n)$. The Dynkin diagrams of the corresponding untwisted affine extensions are represented in Fig.1. The diagrams encode informations about the lengths of the roots $\vec{\alpha}_{i}^{2}$ and their inner products, defined as $\vec{\alpha}_{i} \cdot \vec{\alpha}_{j}=\sum_{k=1}^{r} \alpha_{i}^{k} \alpha_{j}^{k}$ (no complex conjugation). The Kač labels are also explicitly shown, with $q_{0}=1$ so that $\sum_{i=0}^{r} q_{i} \vec{\alpha}_{i}=0$. Their sum defines the Coxeter number $h$

$$
h=\sum_{i=0}^{r} q_{i}
$$

1) $D(n+1, n), n \geq 1$

The $D(n+1, n)$ superalgebra is a simple superalgebra with rank $2 n+1$. An explicit realization is given in terms of matrices in $\mathcal{M}(2 n+2 \mid 2 n ; \mathcal{C})$ satisfying the condition

$$
M^{\text {st }} K+(-1)^{\operatorname{deg}(M)} K M=0
$$


where $M^{\text {st }}$ indicates the supertranspose of the matrix $M$ and

$$
K=\left(\begin{array}{cccc}
0 & \mathbf{I}_{n+1} & 0 & 0 \\
\mathbf{I}_{n+1} & 0 & 0 & 0 \\
0 & 0 & 0 & \mathbf{I}_{n} \\
0 & 0 & -\mathbf{I}_{n} & 0
\end{array}\right)
$$

This provides the fundamental representation of the algebra $D(n+1, n)$.

The completely fermionic extended Dynkin diagram is given in Fig.1. A corresponding choice of positive simple roots and generators is

$$
\begin{array}{rlrl}
\vec{\alpha}_{1} & =\vec{\varepsilon}_{1}-\vec{\delta}_{1} & e_{1}^{+}=e_{1,2 n+3}+e_{3 n+3, n+2} \\
\vec{\alpha}_{2} & =\vec{\delta}_{1}-\vec{\varepsilon}_{2} & e_{2}^{+} & =e_{n+3,3 n+3}-e_{2 n+3,2} \\
& \ldots & & \ldots \\
\vec{\alpha}_{2 n-1} & =\vec{\varepsilon}_{n}-\vec{\delta}_{n} & e_{2 n-1}^{+} & =e_{n, 3 n+2}+e_{4 n+2,2 n+1} \\
\vec{\alpha}_{2 n} & =\vec{\delta}_{n}-\vec{\varepsilon}_{n+1} & e_{2 n}^{+} & =e_{2 n+2,4 n+2}-e_{3 n+2, n+1} \\
\vec{\alpha}_{2 n+1} & =\vec{\delta}_{n}+\vec{\varepsilon}_{n+1} & e_{2 n+1}^{+} & =e_{n+1,4 n+2}-e_{3 n+2,2 n+2}
\end{array}
$$

while the lowest root is given by

$$
\vec{\alpha}_{0}=-\vec{\varepsilon}_{1}-\vec{\delta}_{1} \quad e_{0}^{+}=e_{n+2,2 n+3}+e_{3 n+3,1}
$$

For any generator $e_{j}^{+}=e_{a b} \pm e_{c d}$ associated to a positive simple root, the generator associated to the corresponding negative root is $e_{j}^{-}=e_{b a} \mp e_{d c}$. An explicit expression for the roots can be obtained if we choose the $\vec{\varepsilon}$ 's and $\vec{\delta}$ 's as $(2 n+1)$-dimensional vectors of the form

$$
\begin{array}{rlrl}
\left(\vec{\varepsilon}_{j}\right)_{k} & =\delta_{k j} & j & =1, \cdots, n+1 \\
\left(\vec{\delta}_{j}\right)_{k} & =i \delta_{k, j+n+1} & j & =1, \cdots, n
\end{array}
$$

A convenient basis for the Cartan subalgebra is the following:

$$
\begin{aligned}
h_{a} & =e_{a, a}-e_{a+n+1, a+n+1} & & a=1, \cdots, n+1 \\
h_{a} & =i\left(e_{a+n+1, a+n+1}-e_{a+2 n+1, a+2 n+1}\right) & & a=n+2, \cdots, 2 n+1
\end{aligned}
$$

The Weyl vector $\vec{\rho}$ of the algebra can be obtained as half the sum of the vectors $\vec{\nu}_{j}$ dual to the simple roots $\left(\vec{\alpha}_{i} \cdot \vec{\nu}_{j}=\delta_{i j}\right)$. For this case we have

$$
\vec{\rho}=n \vec{\varepsilon}_{1}-\left(n-\frac{1}{2}\right) \vec{\delta}_{1}+(n-1) \vec{\varepsilon}_{2}-\left(n-\frac{3}{2}\right) \vec{\delta}_{2}+\cdots+\vec{\varepsilon}_{n}-\frac{1}{2} \vec{\delta}_{n}
$$


The weights of the fundamental representation can be computed by using the general procedure described above. They are

$$
\begin{aligned}
\vec{\lambda}_{j} & =-\vec{\lambda}_{4 n+3-j}=\sum_{k=j}^{2 n-1} \vec{\alpha}_{k}+\frac{1}{2} \vec{\alpha}_{2 n}+\frac{1}{2} \vec{\alpha}_{2 n+1} \quad j=1, \cdots, 2 n-1 \\
\vec{\lambda}_{2 n} & =-\vec{\lambda}_{2 n+3}=\frac{1}{2} \vec{\alpha}_{2 n}+\frac{1}{2} \vec{\alpha}_{2 n+1} \\
\vec{\lambda}_{2 n+1} & =-\vec{\lambda}_{2 n+2}=-\frac{1}{2} \vec{\alpha}_{2 n}+\frac{1}{2} \vec{\alpha}_{2 n+1}
\end{aligned}
$$

For the $D(n+1, n)$ superalgebra, the ordering of the weights is not unique. We have chosen the above labeling so that the Miura operator can be written in terms of the weights in a manner similar to the $B(n, n)$ and $A(n, n)$ cases (cfr. eqs. 2.15, 2.21, 2.27)).

2) $B(n, n), n \geq 1$

This is a simple superalgebra with rank $2 n$. The fundamental representation is given in terms of matrices in $\mathcal{M}(2 n+1 \mid 2 n ; \mathcal{C})$ satisfying the condition (A.10) where in this case $K$ is given by

$$
K=\left(\begin{array}{ccccc}
0 & \mathbf{I}_{n} & 0 & 0 & 0 \\
\mathbf{I}_{n} & 0 & 0 & 0 & 0 \\
0 & 0 & 1 & 0 & 0 \\
0 & 0 & 0 & 0 & \mathbf{I}_{n} \\
0 & 0 & 0 & -\mathbf{I}_{n} & 0
\end{array}\right)
$$

The fermionic set of simple roots corresponding to the Dynkin diagram in Fig.1 and the generators $e_{j}^{+}$are

$$
\begin{array}{rlrl}
\vec{\alpha}_{1} & =\vec{\varepsilon}_{1}-\vec{\delta}_{1} & e_{1}^{+}=e_{1,2 n+2}+e_{3 n+2, n+1} \\
\vec{\alpha}_{2}=\vec{\delta}_{1}-\vec{\varepsilon}_{2} & e_{2}^{+}=e_{n+2,3 n+2}-e_{2 n+2,2} \\
& \ldots & \cdots \\
\vec{\alpha}_{2 n-2} & =\vec{\delta}_{n-1}-\vec{\varepsilon}_{n} & e_{2 n-2}^{+}=e_{2 n, 4 n}-e_{3 n, n} \\
\vec{\alpha}_{2 n-1}=\vec{\varepsilon}_{n}-\vec{\delta}_{n} & e_{2 n-1}^{+}=e_{n, 3 n+1}+e_{4 n+1,2 n} \\
\vec{\alpha}_{2 n}=\vec{\delta}_{n} & e_{2 n}^{+}=e_{2 n+1,4 n+1}-e_{3 n+1,2 n+1}
\end{array}
$$

We note that in Fig. 1 the Dynkin diagram of the $B(n, n)$ superalgebra can be obtained by folding the corresponding one of the $D(n+1, n)$ series. The untwisted fermionic extension is obtained by adding to the set of simple roots the lowest root

$$
\vec{\alpha}_{0}=-\vec{\varepsilon}_{1}-\vec{\delta}_{1} \quad e_{0}^{+}=e_{n+1,2 n+2}+e_{3 n+2,1}
$$

As for the previous case, the generators for the negative roots are defined as $e_{j}^{-}=e_{b a} \mp e_{d c}$, whenever the corresponding positive root has generator $e_{j}^{+}=e_{a b} \pm e_{c d}$. An explicit 
realization for the $2 n$-dimensional vectors $\vec{\varepsilon}$ 's and $\vec{\delta}$ 's is

$$
\begin{array}{ll}
\left(\vec{\varepsilon}_{j}\right)_{k}=\delta_{k j} & j=1, \cdots, n \\
\left(\vec{\delta}_{j}\right)_{k}=i \delta_{k, j+n} & j=1, \cdots, n
\end{array}
$$

A basis for the Cartan subalgebra is

$$
\begin{array}{ll}
h_{a}=e_{a, a}-e_{a+n, a+n} & a=1, \cdots, n \\
h_{a}=i\left(e_{a+n+1, a+n+1}-e_{a+2 n+1, a+2 n+1}\right) & a=n+1, \cdots, 2 n
\end{array}
$$

Also in this case, the Weyl vector is given by the expression in eq.(A.16).

The calculation of the weights of the fundamental representation gives the following result

$$
\begin{aligned}
\vec{\lambda}_{j} & =-\vec{\lambda}_{4 n+2-j}=\sum_{k=j}^{2 n} \vec{\alpha}_{k} \quad j=1, \cdots, 2 n \\
\vec{\lambda}_{2 n+1} & =0
\end{aligned}
$$

where we have labelled the weights from the highest $\vec{\lambda}_{1}$ to the lowest $\vec{\lambda}_{4 n+1}$.

3) $A(n, n), n \geq 1$

The fundamental representation of $A(n, n)$ is given in terms of matrices with supertrace equal to zero in $\mathcal{M}(n+1 \mid n+1 ; \mathcal{C})$. This superalgebra is not simple since it contains a one-dimensional invariant subalgebra generated by the element $\mathbf{I}_{2 n+2}$. In the following we refer to $A(n, n)$ as the superalgebra obtained by factoring out the invariant subalgebra. This factorization is realized explicitly by identifying any two elements of $A(n, n)$ whenever they differ by a multiple of the identity $\mathbf{I}_{2 n+2}$. The rank of the algebra is $2 n$ but, as a consequence of the fact that the algebra is not simple, the number of simple roots exceeds the rank by one. Only $2 n$ of them are independent, so that they can still be represented by vectors in a $2 n$-dimensional linear space. A realization of the fermionic roots corresponding to the Dynkin diagram in Fig.1 can be given in terms of vectors $\vec{\varepsilon}_{i}$, $\vec{\delta}_{j}$ as in eq.(A.21). We obtain for the roots and corresponding generators

$$
\begin{array}{rlrl}
\vec{\alpha}_{1} & =\vec{\varepsilon}_{1}-\vec{\delta}_{1} & e_{1}^{+} & =\sqrt{2} e_{1, n+2} \\
\vec{\alpha}_{2} & =\vec{\varepsilon}_{1}+\vec{\delta}_{1} & e_{2}^{+} & =\sqrt{2} e_{n+2,2} \\
\vec{\alpha}_{3} & =-\vec{\varepsilon}_{1}+\vec{\delta}_{1}+\vec{\varepsilon}_{2}-\vec{\delta}_{2} & e_{3}^{+} & =\sqrt{2} e_{2, n+3} \\
\vec{\alpha}_{4} & =\vec{\varepsilon}_{2}+\vec{\delta}_{2} & e_{4}^{+}=\sqrt{2} e_{n+3,3} \\
& \ldots & \cdots \\
\vec{\alpha}_{2 n-1} & =-\vec{\varepsilon}_{n-1}+\vec{\delta}_{n-1}+\vec{\varepsilon}_{n}-\vec{\delta}_{n} & e_{2 n-1}^{+}=\sqrt{2} e_{n, 2 n+1} \\
\vec{\alpha}_{2 n} & =\vec{\varepsilon}_{n}+\vec{\delta}_{n} & e_{2 n}^{+} & =\sqrt{2} e_{2 n+1, n+1} \\
\vec{\alpha}_{2 n+1} & =-\vec{\varepsilon}_{n}+\vec{\delta}_{n} & e_{2 n+1}^{+} & =\sqrt{2} e_{n+1,2 n+2}
\end{array}
$$


As noticed above the simple roots are not all independent since $\sum_{j=0}^{n} \vec{\alpha}_{2 j+1}=0$. The fermionic extension is realized by

$$
\vec{\alpha}_{0}=-\sum_{j=1}^{n}\left(\vec{\varepsilon}_{j}+\vec{\delta}_{j}\right) \quad e_{0}^{+}=\sqrt{2} e_{2 n+2,1}
$$

The generators corresponding to negative simple roots are $e_{2 j}^{-}=-\left(e_{2 j}^{+}\right)^{\mathrm{t}}, e_{2 j+1}^{-}=\left(e_{2 j+1}^{+}\right)^{\mathrm{t}}$, $j=0, \cdots, n$. A basis of the Cartan subalgebra is given by the following matrices

$$
\begin{array}{lll}
h_{a}=\sum_{j=1}^{a} e_{j, j}-e_{a+1, a+1}+\sum_{j=2}^{a} e_{n+j, n+j} & a=1, \cdots, n \\
h_{a}=i\left(\sum_{j=1}^{a-n+1} e_{j, j}+\sum_{j=2}^{a-n} e_{n+j, n+j}+2 e_{a+1, a+1}\right) & a=n+1, \cdots, 2 n
\end{array}
$$

where we have identified

$$
e_{2 n+2,2 n+2} \equiv-\sum_{k=1}^{2 n+1} e_{k, k}
$$

as a consequence of the factorization of the invariant subalgebra. The Weyl vector can be defined as half the sum of vectors dual to the first $2 n$ independent simple roots. We obtain

$$
\vec{\rho}=\frac{1}{4} \sum_{k=1}^{n}\left[(k+1) \vec{\varepsilon}_{k}+(k-1) \vec{\delta}_{k}\right]
$$

Finally the weights of the fundamental representation, with the unique ordering from the highest to the lowest one, are

$$
\begin{aligned}
\vec{\lambda}_{j} & =\sum_{k=j}^{2 n+1} \vec{\alpha}_{k} \quad j=1, \cdots, 2 n+1 \\
\vec{\lambda}_{2 n+2} & =0
\end{aligned}
$$




\section{References}

[1] P.P. Kulish and E.R. Nisimov, Theor. Math. Phys. 29 (1976), 922;

R. Flume, Phys. Lett. 62B (1976), 93; Phys. Lett. 68B (1977) 487;

J.H. Lowenstein and E.R. Speer, Comm. Math. Phys. 63 (1978), 97;

R. Sasaki and I. Yamanaka, Adv. Stud. Pure Math. 16 (1988), 271; Progr. Theor. Phys. 79 (1988), 1167;

T. Marinucci and S. Sciuto, Nucl. Phys. B156 (1979), 144;

E.R. Nisimov, Nucl. Phys. B163 (1980), 374;

A.B. Zamolodchikov, Int. J. Mod. Phys. A3 (1988), 743; Rutherford preprint RAL89-001.

[2] L. Palla, Nucl. Phys. B341 (1990) 714.

[3] G.W. Delius, M.T. Grisaru and D. Zanon, "Quantum conserved currents in affine Toda theories", IFUM 412/FT (1992), to be published in Nucl. Phys. B.

[4] A.B. Zamolodchikov and Al.B. Zamolodchikov, Ann. Phys. (NY) 120 (1979) 253.

[5] A.E. Arinshtein, V.A. Fateev and A.B. Zamolodchikov, Phys. Lett. 87B (1979) 389;

J.L. Cardy and G. Mussardo, Phys. Lett. 225B (1989) 275;

P.G.O. Freund, T.R. Klassen and E. Melzer, Phys. Lett. 229B (1989) 243;

C. Destri and H.J. de Vega, Phys. Lett. 233B (1989) 336;

P. Christe and G. Mussardo, Nucl. Phys. B330 (1990) 465; Int. J. Mod. Phys. A5 (1990) 4581;

T.R. Klassen and E. Melzer, Nucl. Phys. B338 (1990) 485;

H.W. Braden, E. Corrigan, P.E. Dorey and R. Sasaki, Nucl. Phys. B338 (1990) 689;

G.W. Delius, M.T. Grisaru, S. Penati and D. Zanon, Phys. Lett. 256B (1991) 164;

Nucl. Phys. B359 (1991) 125;

C. Destri, H.J. de Vega and V.A. Fateev, Phys. Lett. 256B (1991) 173.

[6] G.W. Delius, M.T. Grisaru and D. Zanon, Phys. Lett. 277B (1992) 414; "Exact S-matrices for nonsimply-laced affine Toda theories", IFUM 413/FT (1992), to be published in Nucl. Phys. B.

[7] M. A. Olshanetsky, Comm. Math. Phys. 88 (1983) 63.

[8] V.G. Kač, Adv. Math. 26 (1977) 8; Adv. Math. 30 (1978) 85.

[9] D.A. Leites, M.V. Saviliev and V.V. Serganova, in "Group theoretical methods in physics", VNU Science Press (1986);

L. Frappat, A. Sciarrino and P. Sorba, Comm. Math. Phys. 121 (1989) 457. 
[10] J. Evans and T. Hollowood, Nucl. Phys. B352 (1991) 723.

[11] A.V. Mikhailov, M.A. Olshanetsky and A.M. Perelomov, Comm. Math. Phys. 79 (1981) 473.

[12] V.A. Fateev and S.I. Lukyanov, "Additional symmetries and exactly soluble models in two-dimensional conformal field theory", I-III, Kiev/Moscow preprints (1988/89).

[13] P. Mansfield and B. Spence, Nucl. Phys. B362 (1991) 294.

[14] S. Komata, K. Mohri and H. Nohara, Nucl. Phys. B359 (1991) 168;

P. Mansfield and B. Spence, Phys. Lett. 265B (1991) 79.

[15] S. Penati and D. Zanon, "Supersymmetric, integrable Toda field theories: the $B(1,1)$ model", preprint IFUM 421/FT (1992), to be published in Phys. Lett. B.

[16] T. Inami and H. Kanno, Comm. Math. Phys. 136 (1991) 519.

[17] M.T. Grisaru, S. Penati and D. Zanon, Nucl. Phys. B369 (1992) 373.

[18] P. di Vecchia and S. Ferrara, Nucl. Phys. B130 (1977) 93;

C. Imbimbo and S. Mukhi, Nucl. Phys. B247 (1984) 471.

[19] G.W. Delius, M.T. Grisaru. S. Penati and D. Zanon, work in progress.

[20] J.F. Cornwell, "Group theory in Physics" vol. III, Academic Press (1989). 


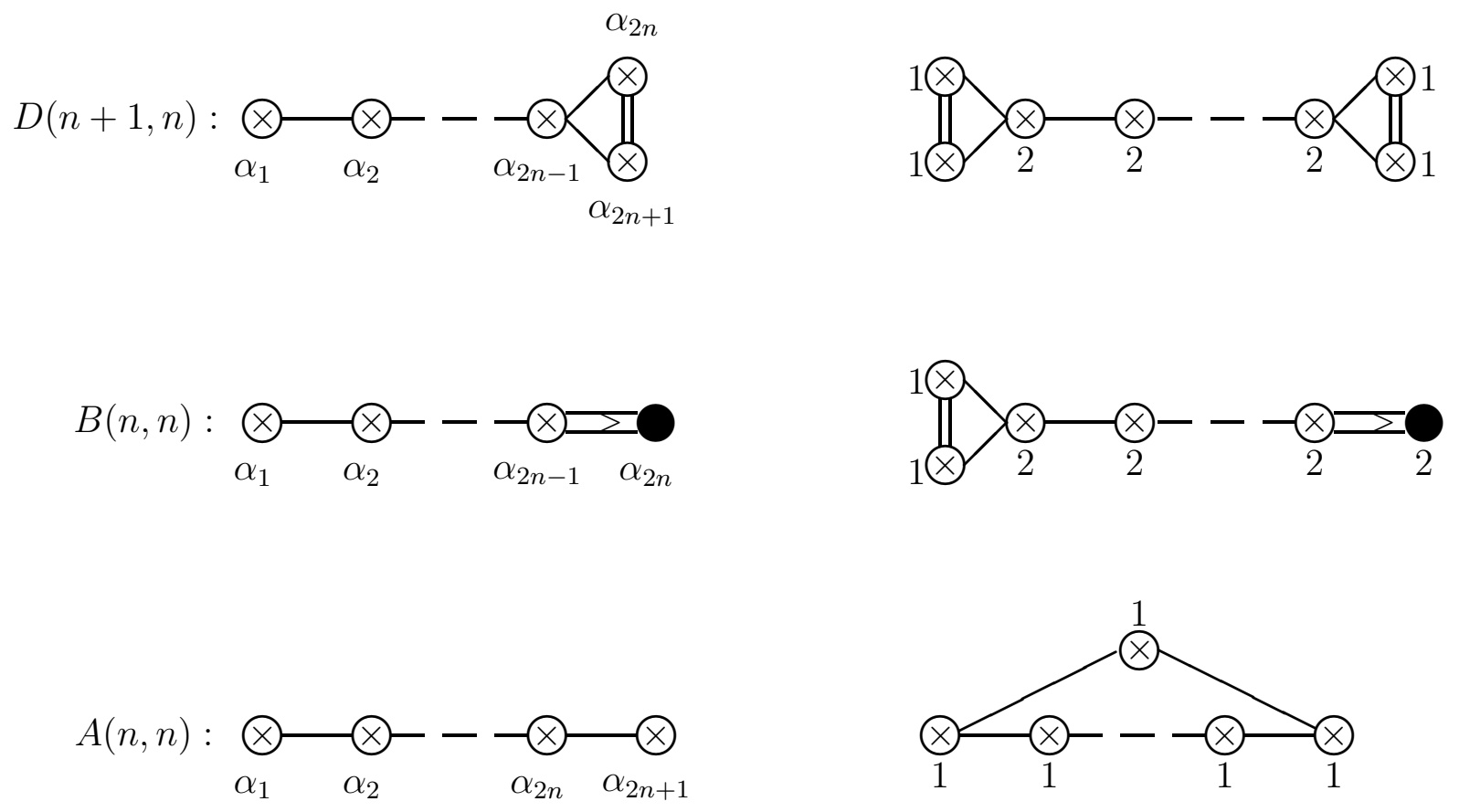

Figure 1: Fermionic Dynkin diagrams for Lie superalgebras and their affine extensions. The crossed circles denote fermionic roots with vanishing norm. The numbers in the affine Dynkin diagrams are the Kač labels. 


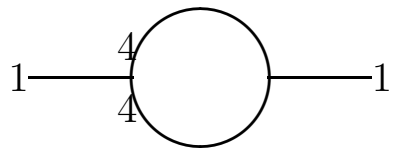

a)

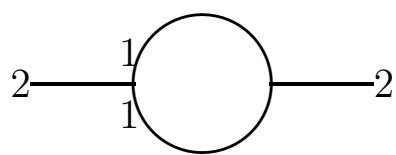

d)

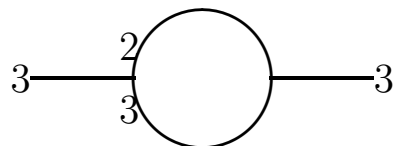

h)

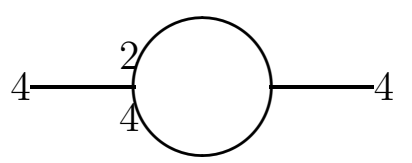

$\mathrm{m})$

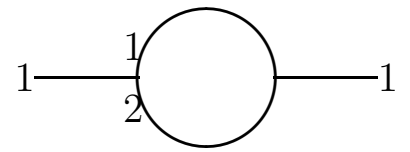

b)

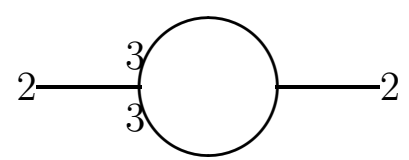

e)

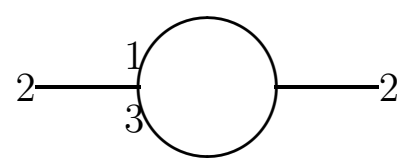

g)

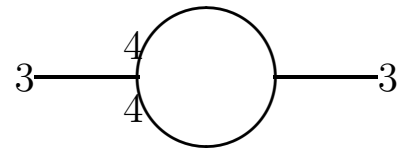

i)

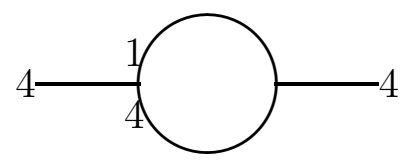

n)

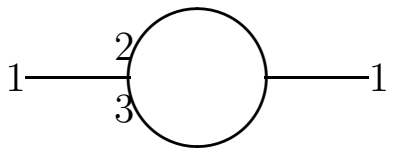

c)

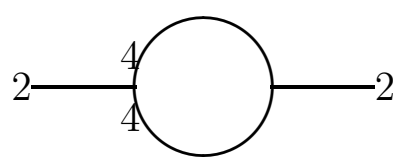

f)

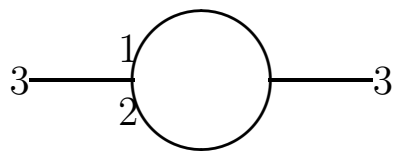

l)

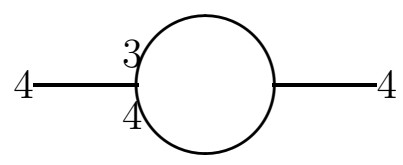

o)

Figure 2: Diagrams of one-loop mass corrections for the $B^{(1)}(2,2)$ theory. The numbers denote the various particles. 